ESAIM: COCV 19 (2013) 947-975

DOI: $10.1051 / \mathrm{cocv} / 2012040$
ESAIM: Control, Optimisation and Calculus of Variations

www.esaim-cocv.org

\title{
TWO-INPUT CONTROL SYSTEMS ON THE EUCLIDEAN GROUP SE (2)
}

\author{
Ross M. Adams ${ }^{1}$, Rory Biggs ${ }^{1}$ and Claudiu C. Remsing ${ }^{1}$
}

\begin{abstract}
Any two-input left-invariant control affine system of full rank, evolving on the Euclidean group SE (2), is (detached) feedback equivalent to one of three typical cases. In each case, we consider an optimal control problem which is then lifted, via the Pontryagin Maximum Principle, to a Hamiltonian system on the dual space $\mathfrak{s e}(2)^{*}$. These reduced Hamilton-Poisson systems are the main topic of this paper. A qualitative analysis of each reduced system is performed. This analysis includes a study of the stability nature of all equilibrium states, as well as qualitative descriptions of all integral curves. Finally, the reduced Hamilton equations are explicitly integrated by Jacobi elliptic functions. Parametrisations for all integral curves are exhibited.
\end{abstract}

Mathematics Subject Classification. 49J15, 93D05, 22E60, 53D17.

Received September 22, 2011. Revised June 4, 2012.

Published online July 4, 2013.

\section{INTRODUCTION}

A general left-invariant control affine system on the Euclidean group SE (2) has the form $\dot{g}=g\left(A+u_{1} B_{1}+\right.$ $\cdots+u_{\ell} B_{\ell}$ ), where $A, B_{1}, \ldots, B_{\ell} \in \mathfrak{s e}(2), 1 \leq \ell \leq 3$. (The elements $B_{1}, \ldots, B_{\ell}$ are assumed to be linearly independent). Specific left-invariant optimal control problems on the Euclidean group SE (2), associated with the above mentioned control systems, have been studied by several authors (see, e.g., $[2,10-12,17,22,23,25-28]$ ).

In this paper, we consider only two-input control systems, i.e., systems of the form $\dot{g}=g\left(A+u_{1} B_{1}+u_{2} B_{2}\right)$. Any such homogeneous full-rank control system is (detached feedback) equivalent to the control system $\Sigma_{0}$ : $\dot{g}=g\left(u_{1} E_{2}+u_{2} E_{3}\right)$. Then again, any such inhomogeneous control system is (detached feedback) equivalent to exactly one of the control systems $\Sigma_{1}: \dot{g}=g\left(E_{1}+u_{1} E_{2}+u_{2} E_{3}\right)$ and $\Sigma_{2, \alpha}: \dot{g}=g\left(\alpha E_{3}+u_{1} E_{1}+u_{2} E_{2}\right)$, $\alpha>0$. Here $E_{1}, E_{2}$ and $E_{3}$ denote elements of the standard basis for $\mathfrak{s e}(2)$. In each typical case, we consider an optimal control problem (with quadratic cost) of the form

$$
\begin{gathered}
\dot{g}=g\left(A+u_{1} B_{1}+u_{2} B_{2}\right), \quad g \in \mathrm{SE}(2), u=\left(u_{1}, u_{2}\right) \in \mathbb{R}^{2} \\
g(0)=g_{0}, \quad g(T)=g_{T} \\
\mathcal{J}=\frac{1}{2} \int_{0}^{T}\left(c_{1} u_{1}^{2}(t)+c_{2} u_{2}^{2}(t)\right) \mathrm{d} t \rightarrow \min .
\end{gathered}
$$

\footnotetext{
Keywords and phrases. Left-invariant control system, (detached) feedback equivalence, Lie-Poisson structure, energy-Casimir method, Jacobi elliptic function.

1 Department of Mathematics (Pure and Applied), Rhodes University, Grahamstown, South Africa. dros@webmail.co.za; rorybiggs@gmail.com; c.c.remsing@ru.ac.za
} 
Each problem is lifted, via the Pontryagin Maximum Principle, to a Hamiltonian system on the dual space $\mathfrak{s e}(2)^{*}$. Then the (minus) Lie-Poisson structure on $\mathfrak{s e}(2)^{*}$ is used to derive the equations for extrema (cf. $[3,11,14])$. The stability nature of all equilibrium states for the reduced system is then investigated by the energy-Casimir method. Also, a qualitative description of all integral curves of the reduced system is given. Finally, these equations are explicitly integrated by Jacobi elliptic functions. A brief description of this process is given now.

First, we partition the set of initial conditions in terms of simple inequalities. Specifically, we distinguish between various ways that the level sets, defined by the constants of motion, intersect. When required, this set is further partitioned in order to facilitate integration. This enables one to distinguish between solution curves with different explicit expressions. In each case, the extremal equations are reduced to a (separable) differential equation and then transformed into standard form (see, e.g., [4] or [15]). Thereafter, an integral formula is applied. Consequently, by use of the constants of motion (and allowing for possible changes in sign), an explicit expression for the solution is obtained.

The paper is organized as follows. In Section 2 we review some basic facts regarding left-invariant control systems, optimal control, the energy-Casimir method and Jacobi elliptic functions. In Section 3 we classify all two-input left-invariant control affine systems on SE (2) and then introduce a general optimal control problem (with quadratic cost) to be considered for each equivalence class. In Section 4 a qualitative analysis of the reduced Hamiltonian systems is given and in Section 5 the reduced systems are explicitly integrated. A tabulation of integral curves is included as an appendix. We conclude the paper with a summary and a few remarks.

\section{Preliminaries}

\subsection{Invariant control systems and optimal control}

Invariant control systems on Lie groups were first considered in 1972 by Brockett [8] and by Jurdjevic and Sussmann [13]. A left-invariant control system $\Sigma$ is a (smooth) control system evolving on a (real, finitedimensional) Lie group $\mathrm{G}$, whose dynamics $\Xi: \mathrm{G} \times U \rightarrow T \mathrm{G}$ are invariant under left translations. (The tangent bundle $T G$ is identified with $G \times \mathfrak{g}$, where $\mathfrak{g}$ is the Lie algebra of $\mathrm{G}$ ). For the sake of convenience, we shall assume that $G$ is a matrix Lie group. Also, for the purposes of this paper, we may assume that $U=\mathbb{R}^{\ell}$. Such a control system is described as follows ( $c f .[3,11,24])$

$$
\dot{g}=\Xi(g, u), \quad g \in \mathrm{G}, u \in \mathbb{R}^{\ell}
$$

where $\Xi(g, u)=g \Xi(\mathbf{1}, u) \in T_{g} \mathrm{G}$.

Admissible controls are bounded and measurable maps $u(\cdot):[0, T] \rightarrow \mathbb{R}^{\ell}$, whereas the parametrisation map $\Xi(\mathbf{1}, \cdot): \mathbb{R}^{\ell} \rightarrow \mathfrak{g}$ is an embedding. The trace $\Gamma=\operatorname{im} \Xi(\mathbf{1}, \cdot)$ is a submanifold of $\mathfrak{g}$ so that $\Gamma=$ $\left\{\Xi_{u}=\Xi(\mathbf{1}, u): u \in \mathbb{R}^{\ell}\right\}(c f$. [5,6]). A left-invariant control affine system is one whose parametrisation map is affine. For such a system, the trace $\Gamma$ is an affine subspace of $\mathfrak{g}$. We say that the system has full rank if the Lie algebra generated by its trace, Lie $(\Gamma)$, coincides with $\mathfrak{g}$. A trajectory for an admissible control $u(\cdot):[0, T] \rightarrow \mathbb{R}^{\ell}$ is an absolutely continuous curve $g(\cdot):[0, T] \rightarrow \mathrm{G}$ such that $\dot{g}(t)=g(t) \Xi(\mathbf{1}, u(t))$ for almost every $t \in[0, T]$.

We shall denote a (left-invariant control) system $\Sigma$ by $(\mathrm{G}, \Xi)$ (see, e.g., [5,6]). We say that a system $\Sigma=(\mathrm{G}, \Xi)$ is connected if its state space $\mathrm{G}$ is connected. Let $\Sigma=(\mathrm{G}, \Xi)$ and $\Sigma^{\prime}=\left(\mathrm{G}^{\prime}, \Xi^{\prime}\right)$ be two connected full-rank systems with traces $\Gamma \subseteq \mathfrak{g}$ and $\Gamma^{\prime} \subseteq \mathfrak{g}^{\prime}$, respectively. We say that $\Sigma$ and $\Sigma^{\prime}$ are locally detached feedback equivalent if there exist open neighbourhoods $N$ and $N^{\prime}$ of (the unit elements) $\mathbf{1}$ and $\mathbf{1}^{\prime}$, respectively, and a diffeomorphism $\Phi=\phi \times \varphi: N \times \mathbb{R}^{\ell} \rightarrow N^{\prime} \times \mathbb{R}^{\ell}$ such that $\phi(\mathbf{1})=\mathbf{1}^{\prime}$ and $T_{g} \phi \cdot \Xi(g, u)=\Xi^{\prime}(\phi(g), \varphi(u))$ for $g \in N$ and $u \in \mathbb{R}^{\ell}$. Two detached feedback equivalent systems have the same trajectories (up to a diffeomorphism in the state space), which are parametrised differently by admissible controls. We recall the following result.

Proposition $2.1([6]) . \Sigma=(\mathrm{G}, \Xi)$ and $\Sigma^{\prime}=\left(\mathrm{G}^{\prime}, \Xi^{\prime}\right)$ are locally detached feedback equivalent if and only if there exists a Lie algebra isomorphism $\psi: \mathfrak{g} \rightarrow \mathfrak{g}^{\prime}$ such that $\psi \cdot \Gamma=\Gamma^{\prime}$. 
Now, consider an optimal control problem given by the specification of (i) a left-invariant control system $\Sigma=(\mathrm{G}, \Xi)$, (ii) a cost function $L: \mathbb{R}^{\ell} \rightarrow \mathbb{R}$, and (iii) boundary data, consisting of an initial state $g_{0} \in \mathrm{G}$, a target state $g_{1} \in \mathrm{G}$ and a terminal time $T>0$. Explicitly, we want to minimize the functional $\mathcal{J}=\int_{0}^{T} L(u(t)) \mathrm{d} t$ over the trajectory-control pairs of $\Sigma$ subject to the boundary conditions

$$
g(0)=g_{0}, \quad g(T)=g_{1} .
$$

The Pontryagin Maximum Principle is a necessary condition for optimality which is most naturally expressed in the language of the geometry of the cotangent bundle $T^{*} \mathrm{G}$ of $\mathrm{G}(c f .[3,11])$. The cotangent bundle $T^{*} \mathrm{G}$ can be trivialized (from the left) such that $T^{*} \mathbf{G}=\mathbf{G} \times \mathfrak{g}^{*}$, where $\mathfrak{g}^{*}$ is the dual space of the Lie algebra $\mathfrak{g}$. The dual space $\mathfrak{g}^{*}$ has a natural Poisson structure, called the "minus Lie-Poisson structure", given by

$$
\{F, G\}(p)=-p([\mathrm{~d} F(p), \mathrm{d} G(p)])
$$

for $p \in \mathfrak{g}^{*}$ and $F, G \in C^{\infty}\left(\mathfrak{g}^{*}\right)$. (Note that $\mathrm{d} F(p)$ is a linear function on $\mathfrak{g}^{*}$ and so is an element of $\mathfrak{g}$ ). The Poisson space $\left(\mathfrak{g}^{*},\{\cdot, \cdot\}\right)$ is denoted by $\mathfrak{g}_{-}^{*}$. Each left-invariant Hamiltonian on the cotangent bundle $T^{*} G$ is identified with its reduction on the dual space $\mathfrak{g}_{-}^{*}$. To an optimal control problem (with fixed terminal time)

$$
\int_{0}^{T} L(u(t)) \mathrm{d} t \rightarrow \min
$$

subject to (2.1) and (2.2), we associate, for each real number $\lambda$ and each control parameter $u \in \mathbb{R}^{\ell}$, a Hamiltonian function on $T^{*} \mathrm{G}=\mathrm{G} \times \mathfrak{g}^{*}$ :

$$
\begin{aligned}
H_{u}^{\lambda}(\xi) & =\lambda L(u)+\xi(g \Xi(\mathbf{1}, u)) \\
& =\lambda L(u)+p(\Xi(\mathbf{1}, u)), \quad \xi=(g, p) \in T^{*} \mathrm{G} .
\end{aligned}
$$

The Maximum Principle can be stated, in terms of the above Hamiltonians, as follows.

Maximum Principle. Suppose the trajectory-control pair $(\bar{g}(\cdot), \bar{u}(\cdot))$ defined over the interval $[0, T]$ is a solution for the optimal control problem (2.1)-(2.3). Then, there exists a curve $\xi(\cdot):[0, T] \rightarrow T^{*} G$ with $\xi(t) \in T_{\bar{g}(t)}^{*} \mathrm{G}, t \in[0, T]$, and a real number $\lambda \leq 0$, such that the following conditions hold for almost every $t \in[0, T]:$

$$
\begin{gathered}
(\lambda, \xi(t)) \not \equiv(0,0) \\
\dot{\xi}(t)=\vec{H}_{\bar{u}(t)}^{\lambda}(\xi(t)) \\
H_{\bar{u}(t)}^{\lambda}(\xi(t))=\max _{u} H_{u}^{\lambda}(\xi(t))=\text { const. }
\end{gathered}
$$

An optimal trajectory $\bar{g}(\cdot):[0, T] \rightarrow \mathrm{G}$ is the projection of an integral curve $\xi(\cdot)$ of the (time-varying) Hamiltonian vector field $\vec{H}_{\bar{u}(t)}^{\lambda}$ defined for all $t \in[0, T]$. A trajectory-control pair $(\xi(\cdot), u(\cdot))$ defined on $[0, T]$ is said to be an extremal pair if $\xi(\cdot)$ satisfies the conditions (2.4), (2.5) and (2.6). The projection $\xi(\cdot)$ of an extremal pair is called an extremal. An extremal curve is called normal if $\lambda=-1$ and abnormal if $\lambda=0$. In this paper, we shall be concerned only with normal extremals. Suppose the maximum condition (2.6) eliminates the parameter $u$ from the family of Hamiltonians $\left(H_{u}\right)$, and as a result of this elimination, we obtain a smooth function $H$ (without parameters) on $T^{*} \mathrm{G}$ (in fact, on $\mathfrak{g}_{-}^{*}$ ). Then the whole (left-invariant) optimal control problem reduces to the study of integral curves of a fixed Hamiltonian vector field $\vec{H}$.

\subsection{The energy-Casimir method}

The energy-Casimir method [9] gives sufficient conditions for Lyapunov stability of equilibrium states for certain types of Hamilton-Poisson dynamical systems ( $c f$. $[16,21])$. The method is restricted to certain types of systems, since its implementation relies on an abundant supply of Casimir functions. 
The standard energy-Casimir method states that if $z_{e}$ is an equilibrium point of a Hamiltonian vector field $\vec{H}$ (associated with an energy function $H$ ) and if there exists a Casimir function $C$ such that $z_{e}$ is a critical point of $H+C$ and $d^{2}(H+C)\left(z_{e}\right)$ is (positive or negative) definite, then $z_{e}$ is Lyapunov stable.

Ortega and Ratiu have obtained a generalisation of the standard energy-Casimir method (cf. [18, 19]). This extended version states that if $C=\lambda_{1} C_{1}+\cdots+\lambda_{k} C_{k}$, where $\lambda_{1}, \ldots, \lambda_{k} \in \mathbb{R}$ and $C_{1}, \ldots, C_{k}$ are conserved quantities (i.e., they Poisson commute with the energy function $H$ ), then definiteness of $d^{2}\left(\lambda_{0} H+C\right)\left(z_{e}\right), \lambda_{0} \in$ $\mathbb{R}$ is only required on the intersection (subspace) $W=\operatorname{ker} \mathrm{d} H\left(z_{e}\right) \cap \operatorname{ker} \mathrm{d} C_{1}\left(z_{e}\right) \cap \cdots \cap \operatorname{ker} \mathrm{d} C_{k}\left(z_{e}\right)$.

\subsection{Jacobi elliptic functions}

Given the modulus $k \in[0,1]$, the basic Jacobi elliptic functions $\operatorname{sn}(\cdot, k), \operatorname{cn}(\cdot, k)$ and $\operatorname{dn}(\cdot, k)$ can be defined as

$$
\begin{aligned}
& \operatorname{sn}(x, k)=\sin \operatorname{am}(x, k) \\
& \operatorname{cn}(x, k)=\cos \operatorname{am}(x, k) \\
& \operatorname{dn}(x, k)=\sqrt{1-k^{2} \sin ^{2} \operatorname{am}(x, k)}
\end{aligned}
$$

where $\operatorname{am}(\cdot, k)=F(\cdot, k)^{-1}$ is the amplitude and $F(\varphi, k)=\int_{0}^{\varphi} \frac{\mathrm{d} t}{\sqrt{1-k^{2} \sin ^{2} t}} \cdot$ (For the degenerate cases $k=0$ and $k=1$, we recover the circular functions and the hyperbolic functions, respectively). The complementary modulus $k^{\prime}$ and the number $K$ are then defined as $k^{\prime}=\sqrt{1-k^{2}}$ and $K=F\left(\frac{\pi}{2}, k\right)$. (The functions $\operatorname{sn}(\cdot, k)$ and $\operatorname{cn}(\cdot, k)$ are $4 K$ periodic, whereas $\operatorname{dn}(\cdot, k)$ is $2 K$ periodic). Nine other elliptic functions are defined by taking reciprocals and quotients; in particular, we get $\operatorname{nd}(\cdot, k)=\frac{1}{\operatorname{dn}(\cdot, k)}, \operatorname{sd}(\cdot, k)=\frac{\operatorname{sn}(\cdot, k)}{\operatorname{dn}(\cdot, k)}$ and $\operatorname{cd}(\cdot, k)=\frac{\mathrm{cn}(\cdot, k)}{\operatorname{dn}(\cdot, k)} \cdot$ Simple elliptic integrals can be expressed in terms of appropriate inverse (elliptic) functions. The following formulas hold true (see [4] or [15]):

$$
\begin{array}{ll}
\int_{0}^{x} \frac{\mathrm{d} t}{\sqrt{\left(a^{2}-t^{2}\right)\left(b^{2}-t^{2}\right)}}=\frac{1}{a} \operatorname{sn}^{-1}\left(\frac{1}{b} x, \frac{b}{a}\right), & 0 \leq x \leq b<a \\
\int_{0}^{x} \frac{\mathrm{d} t}{\sqrt{\left(a^{2}+t^{2}\right)\left(b^{2}-t^{2}\right)}}=\frac{1}{\sqrt{a^{2}+b^{2}}} \mathrm{sd}^{-1}\left(\frac{\sqrt{a^{2}+b^{2}}}{a b} x, \frac{b}{\sqrt{a^{2}+b^{2}}}\right), & 0 \leq x \leq b \\
\int_{x}^{a} \frac{\mathrm{d} t}{\sqrt{\left(a^{2}-t^{2}\right)\left(t^{2}-b^{2}\right)}}=\frac{1}{a} \operatorname{dn}^{-1}\left(\frac{1}{a} x, \frac{\sqrt{a^{2}-b^{2}}}{a}\right), & b \leq x \leq a .
\end{array}
$$

\section{Control Systems on SE $(2)$}

We consider two-input left-invariant control affine systems on SE (2). Such a system is fully specified by its parametrisation map $\Xi(\mathbf{1}, u)=A+u_{1} B_{1}+u_{2} B_{2}$. A system is said to be homogeneous if $A \in\left\langle B_{1}, B_{2}\right\rangle$, i.e., the trace $\Gamma$ is a linear subspace of $\mathfrak{s e}(2)$. (In this paper, the notation $\langle\cdot, \cdot\rangle$ is used for the linear span of two vectors). Otherwise, the system is said to be inhomogeneous. A classification of all full-rank two-input systems, under detached feedback equivalence, is provided. We then introduce a general optimal control problem (with diagonal cost) to be considered for each equivalence class.

\subsection{The Euclidean group SE (2)}

The Euclidean group

$$
\mathrm{SE}(2)=\left\{\left[\begin{array}{ll}
1 & 0 \\
\mathbf{v} & R
\end{array}\right]: \mathbf{v} \in \mathbb{R}^{2 \times 1}, R \in \mathrm{SO}(2)\right\}
$$


is a (real) three-dimensional connected matrix Lie group. The associated Lie algebra is given by

$$
\mathfrak{s e}(2)=\left\{\left[\begin{array}{ccc}
0 & 0 & 0 \\
x_{1} & 0 & -x_{3} \\
x_{2} & x_{3} & 0
\end{array}\right]: x_{1}, x_{2}, x_{3} \in \mathbb{R}\right\}
$$

Let

$$
E_{1}=\left[\begin{array}{lll}
0 & 0 & 0 \\
1 & 0 & 0 \\
0 & 0 & 0
\end{array}\right], \quad E_{2}=\left[\begin{array}{lll}
0 & 0 & 0 \\
0 & 0 & 0 \\
1 & 0 & 0
\end{array}\right], \quad E_{3}=\left[\begin{array}{ccc}
0 & 0 & 0 \\
0 & 0 & -1 \\
0 & 1 & 0
\end{array}\right]
$$

be the standard basis of $\mathfrak{s e}(2)$. (The bracket operation is given by $\left[E_{2}, E_{3}\right]=E_{1},\left[E_{3}, E_{1}\right]=E_{2}$ and $\left[E_{1}, E_{2}\right]=$ $0)$. With respect to this basis, the group of Lie algebra automorphisms of $\mathfrak{s e}(2)$ is given by

$$
\text { Aut }(\mathfrak{s e}(2))=\left\{\left[\begin{array}{ccc}
x & y & v \\
-\varsigma y & \varsigma x & w \\
0 & 0 & \varsigma
\end{array}\right]: x, y, v, w \in \mathbb{R}, x^{2}+y^{2} \neq 0, \varsigma= \pm 1\right\} .
$$

We use the non-degenerate bilinear form

$$
\left\langle\left\langle\left[\begin{array}{ccc}
0 & 0 & 0 \\
x_{1} & 0 & -x_{3} \\
x_{2} & x_{3} & 0
\end{array}\right],\left[\begin{array}{ccc}
0 & 0 & 0 \\
y_{1} & 0 & -y_{3} \\
y_{2} & y_{3} & 0
\end{array}\right]\right\rangle=x_{1} y_{1}+x_{2} y_{2}+x_{3} y_{3}\right.
$$

to identify $\mathfrak{s e}(2)$ with $\mathfrak{s e}(2)^{*}(c f .[11])$. Then each extremal curve $p(\cdot)$ in $\mathfrak{s e}(2)^{*}$ is identified with a curve $P(\cdot)$ in $\mathfrak{s e}(2)$ via the formula $\langle\langle P(t), X\rangle\rangle=p(t)(X)$ for all $X \in \mathfrak{s e}(2)$. Thus

$$
P(t)=\left[\begin{array}{ccc}
0 & 0 & 0 \\
P_{1}(t) & 0 & -P_{3}(t) \\
P_{2}(t) & P_{3}(t) & 0
\end{array}\right]
$$

where $P_{i}(t)=\left\langle\left\langle P(t), E_{i}\right\rangle\right\rangle=p(t)\left(E_{i}\right)=p_{i}(t), \quad i=1,2,3$.

Now consider a Hamiltonian $H$ on $\mathfrak{s e}(2)_{-}^{*}$. The equations of motion take the following form

$$
\dot{p}_{i}=-p\left(\left[E_{i}, \mathrm{~d} H(p)\right]\right), \quad i=1,2,3
$$

or, explicitly,

$$
\left\{\begin{array}{l}
\dot{p}_{1}=\frac{\partial H}{\partial p_{3}} p_{2} \\
\dot{p}_{2}=-\frac{\partial H}{\partial p_{3}} p_{1} \\
\dot{p}_{3}=\frac{\partial H}{\partial p_{2}} p_{1}-\frac{\partial H}{\partial p_{1}} p_{2}
\end{array}\right.
$$

We note that $C: \mathfrak{s e}(2)_{-}^{*} \rightarrow \mathbb{R}, C(p)=p_{1}^{2}+p_{2}^{2}$ is a Casimir function.

\subsection{Classification of systems}

It turns out that there is only one homogeneous two-input system on SE (2), up to equivalence. Furthermore, in the inhomogeneous case there are only two types. The characterisation of detached feedback equivalence in Proposition 2.1 is used to prove both these results. 
Theorem 3.1. Any full-rank homogeneous two-input system $\Sigma$ is locally detached feedback equivalent to the system $\Sigma_{0}$ with parametrisation

$$
\Xi_{0}(\mathbf{1}, u)=u_{1} E_{2}+u_{2} E_{3} .
$$

Proof. Let the trace of $\Sigma$ be given by $\Gamma=\left\langle\sum_{i=1}^{3} b_{i} E_{i}, \sum_{i=1}^{3} c_{i} E_{i}\right\rangle \cdot$ First, as either $b_{3} \neq 0$ or $c_{3} \neq 0$, we may assume $b_{3} \neq 0$. Then

$$
\Gamma=\left\langle\frac{b_{1}}{b_{3}} E_{1}+\frac{b_{2}}{b_{3}} E_{2}+E_{3},\left(c_{1}-\frac{b_{1} c_{3}}{b_{3}}\right) E_{1}+\left(c_{2}-\frac{b_{2} c_{3}}{b_{3}}\right) E_{2}\right\rangle .
$$

Now let $x=c_{1}-\frac{b_{1} c_{3}}{b_{3}}$ and $y=c_{2}-\frac{b_{2} c_{3}}{b_{3}}$. Then

$$
\psi=\left[\begin{array}{ccc}
y & x & \frac{b_{1}}{b_{3}} \\
-x & y & \frac{b_{2}}{b_{3}} \\
0 & 0 & 1
\end{array}\right]
$$

is a Lie algebra automorphism mapping $\Gamma_{0}=\left\langle E_{2}, E_{3}\right\rangle$ to $\Gamma$.

Theorem 3.2. Any inhomogeneous two-input system $\Sigma$ is locally detached feedback equivalent to exactly one of the following systems: $\Sigma_{1}$ or $\Sigma_{2, \alpha}(\alpha>0)$ with respective parametrisations

$$
\Xi_{1}(\mathbf{1}, u)=E_{1}+u_{1} E_{2}+u_{2} E_{3}, \quad \Xi_{2, \alpha}(\mathbf{1}, u)=\alpha E_{3}+u_{1} E_{1}+u_{2} E_{2} .
$$

Proof. Let the trace of $\Sigma$ be given by

$$
\Gamma=\sum_{i=1}^{3} a_{i} E_{i}+\left\langle\sum_{i=1}^{3} b_{i} E_{i}, \sum_{i=1}^{3} c_{i} E_{i}\right\rangle .
$$

First, consider the case $b_{3} \neq 0$ or $c_{3} \neq 0$. We may assume $b_{3} \neq 0$ and so

$$
\Gamma=a_{1}^{\prime} E_{1}+a_{2}^{\prime} E_{2}+\left\langle b_{1}^{\prime} E_{1}+b_{2}^{\prime} E_{2}+E_{3}, c_{1}^{\prime} E_{1}+c_{2}^{\prime} E_{2}\right\rangle
$$

for some constants $a_{i}^{\prime}, b_{i}^{\prime}, c_{i}^{\prime} \in \mathbb{R}, i=1,2$. Now either $c_{1}^{\prime} \neq 0$ or $c_{2}^{\prime} \neq 0$ and so

$$
\left[\begin{array}{cc}
c_{1}^{\prime} & -c_{2}^{\prime} \\
c_{2}^{\prime} & c_{1}^{\prime}
\end{array}\right]\left[\begin{array}{l}
v_{1} \\
v_{2}
\end{array}\right]=\left[\begin{array}{l}
-a_{1}^{\prime} \\
-a_{2}^{\prime}
\end{array}\right]
$$

has a unique solution. (Note that $v_{2}=0$ leads to a contradiction). Hence

$$
\psi=\left[\begin{array}{ccc}
v_{2} c_{2}^{\prime} & v_{2} c_{1}^{\prime} & b_{1}^{\prime} \\
-v_{2} c_{1}^{\prime} & v_{2} c_{2}^{\prime} & b_{2}^{\prime} \\
0 & 0 & 1
\end{array}\right]
$$

is a Lie algebra automorphism mapping $\Gamma_{1}=E_{1}+\left\langle E_{2}, E_{3}\right\rangle$ to $\Gamma$.

Next, consider the case $b_{3}=0$ and $c_{3}=0$. Then

$$
\Gamma=a_{1} E_{1}+a_{2} E_{2}+a_{3} E_{3}+\left\langle b_{1} E_{1}+b_{2} E_{2}, c_{1} E_{1}+c_{2} E_{2}\right\rangle .
$$

Since $a_{3} \neq 0$ and either $b_{1} \neq 0$ or $b_{2} \neq 0$, we get that

$$
\psi=\left[\begin{array}{ccc}
b_{1} & -\operatorname{sgn}\left(a_{3}\right) b_{2} & \frac{a_{1}}{\alpha} \\
b_{2} & \operatorname{sgn}\left(a_{3}\right) b_{1} & \frac{a_{2}}{\alpha} \\
0 & 0 & \operatorname{sgn}\left(a_{3}\right)
\end{array}\right]
$$

is a Lie algebra automorphism. If we set $\alpha=\left|a_{3}\right|$, then $\psi$ maps $\Gamma_{2, \alpha}=\alpha E_{3}+\left\langle E_{1}, E_{2}\right\rangle$ to $\Gamma$.

Finally, a simple argument shows that $\Sigma_{1}$ is not equivalent to any system $\Sigma_{2, \alpha}$, and that $\Sigma_{2, \alpha}$ is not equivalent to $\Sigma_{2, \beta}$, for any $\alpha \neq \beta, \alpha, \beta>0$. 


\subsection{Left-invariant control problems}

Henceforth, we consider only the systems $\Sigma_{0}, \Sigma_{1}$ and $\Sigma_{2, \alpha}$. In each of these typical cases, we shall investigate the optimal control problem corresponding to an arbitrary diagonal cost $L(u)=c_{1} u_{1}^{2}+c_{2} u_{2}^{2}$, where $c_{1}, c_{2}>0$. Specifically, we shall consider the left-invariant control problems:

$$
\left.\begin{array}{c}
\dot{g}=g\left(u_{1} E_{2}+u_{2} E_{3}\right) \\
g(0)=g_{0}, \quad g(T)=g_{T} \\
\mathcal{J}=\frac{1}{2} \int_{0}^{T}\left(c_{1} u_{1}^{2}(t)+c_{2} u_{2}^{2}(t)\right) \mathrm{d} t \rightarrow \min
\end{array}\right\}
$$

and

$$
\left.\begin{array}{c}
\dot{g}=g\left(\alpha E_{3}+u_{1} E_{1}+u_{2} E_{2}\right) \\
g(0)=g_{0}, \quad g(T)=g_{T} \\
\mathcal{J}=\frac{1}{2} \int_{0}^{T}\left(c_{1} u_{1}^{2}(t)+c_{2} u_{2}^{2}(t)\right) \mathrm{d} t \rightarrow \min .
\end{array}\right\}
$$

Remark 3.3. Each member of a significant subclass of left-invariant control problems on SE (2) is equivalent to one of the above three problems, up to cost-equivalence [7]. (If two cost-extended systems are cost-equivalent, then they have the same extremal trajectories, up to a Lie group isomorphism between their state spaces. The corresponding controls are mapped by an affine isomorphism). More specifically, any full-rank cost-extended system

is cost-equivalent to

$$
\Xi(\mathbf{1}, u)=u_{1} B_{1}+u_{2} B_{2}, \quad L(u)=u^{\top} Q u
$$

$$
\Xi_{0}(\mathbf{1}, u)=u_{1} E_{2}+u_{2} E_{3}, \quad L_{0}(u)=u_{1}^{2}+u_{2}^{2} .
$$

(Here $Q \in \mathbb{R}^{2 \times 2}, Q$ is positive definite; a proof can be found in [7]). LiCP(1) corresponds to (3.1). On the other hand, any cost-extended system

$$
\Xi(\mathbf{1}, u)=A+u_{1} B_{1}+u_{2} B_{2}, \quad L(u)=(u-\mu)^{\top} Q(u-\mu)
$$

where $A \notin\left\langle B_{1}, B_{2}\right\rangle$ is cost-equivalent to one of the cost-extended systems

$$
\begin{aligned}
\Xi_{1}(\mathbf{1}, u) & =E_{1}+u_{1} E_{2}+u_{2} E_{3}, & & L_{1, \beta_{1}}(u)=\left(u_{1}-\mu_{1}\right)^{2}+\beta_{1}\left(u_{2}-\mu_{2}\right)^{2} \\
\Xi_{2, \alpha}(\mathbf{1}, u) & =\alpha E_{3}+u_{1} E_{1}+u_{2} E_{2}, & & L_{2, \beta_{2}}(u)=u_{1}^{2}+\beta_{2} u_{2}^{2}
\end{aligned}
$$

where $\alpha, \beta_{1}>0$ and $\beta_{2} \geq 1$. (Here $\mu \in \mathbb{R}^{2}, Q \in \mathbb{R}^{2 \times 2}$, and $Q$ is positive definite). $\operatorname{LiCP}(2)$ corresponds to (3.2) with $\mu_{1}=\mu_{2}=0$, whereas $\operatorname{LiCP}(3)$ corresponds to (3.3).

The following three results easily follow.

Proposition 3.4. For the $\mathrm{LiCP}(1)$, the (normal) extremal control is given by $u_{1}=\frac{1}{c_{1}} p_{2}, u_{2}=\frac{1}{c_{2}} p_{3}$, where $H_{1}(p)=\frac{1}{2}\left(\frac{1}{c_{1}} p_{2}^{2}+\frac{1}{c_{2}} p_{3}^{2}\right)$ and

$$
\left\{\begin{array}{l}
\dot{p}_{1}=\frac{1}{c_{2}} p_{2} p_{3} \\
\dot{p}_{2}=-\frac{1}{c_{2}} p_{1} p_{3} \\
\dot{p}_{3}=\frac{1}{c_{1}} p_{1} p_{2} .
\end{array}\right.
$$


Proposition 3.5. For the $\operatorname{LiCP}(2)$, the (normal) extremal control is given by $u_{1}=\frac{1}{c_{1}} p_{2}, u_{2}=\frac{1}{c_{2}} p_{3}$, where $H_{2}(p)=p_{1}+\frac{1}{2}\left(\frac{1}{c_{1}} p_{2}^{2}+\frac{1}{c_{2}} p_{3}^{2}\right)$ and

$$
\left\{\begin{array}{l}
\dot{p}_{1}=\frac{1}{c_{2}} p_{2} p_{3} \\
\dot{p}_{2}=-\frac{1}{c_{2}} p_{1} p_{3} \\
\dot{p}_{3}=\left(\frac{1}{c_{1}} p_{1}-1\right) p_{2} .
\end{array}\right.
$$

Proposition 3.6. For the $\operatorname{LiCP}(3)$, the (normal) extremal control is given by $u_{1}=\frac{1}{c_{1}} p_{1}, u_{2}=\frac{1}{c_{2}} p_{2}$, where $H_{3}(p)=\alpha p_{3}+\frac{1}{2}\left(\frac{1}{c_{1}} p_{1}^{2}+\frac{1}{c_{2}} p_{2}^{2}\right)$ and

$$
\left\{\begin{array}{l}
\dot{p}_{1}=\alpha p_{2} \\
\dot{p}_{2}=-\alpha p_{1} \\
\dot{p}_{3}=\left(\frac{1}{c_{2}}-\frac{1}{c_{1}}\right) p_{1} p_{2} .
\end{array}\right.
$$

\section{Qualitative Analysis}

In this section a qualitative analysis of the reduced Hamilton-Poisson systems (3.4)-(3.6) is performed. The stability nature of every equilibrium state is determined. The vector fields $\vec{H}_{1}, \vec{H}_{2}$, and $\vec{H}_{3}$ are shown to be complete. Subsequently, each maximal integral curve is described as a constant, periodic or bounded curve.

\subsection{Equilibrium states}

The equilibrium states for (3.4) are

$$
e_{1}^{\mu}=(\mu, 0,0), \quad e_{2}^{\nu}=(0, \nu, 0) \quad \text { and } \quad e_{3}^{\mu}=(0,0, \mu)
$$

where $\mu, \nu \in \mathbb{R}, \nu \neq 0$.

Theorem 4.1. The equilibrium states have the following behaviour:

(i) Each equilibrium state $e_{1}^{\mu}$ is stable.

(ii) Each equilibrium state $e_{2}^{\nu}$ is unstable.

(iii) Each equilibrium state $e_{3}^{\mu}$ is stable.

Proof. The linearization of the system is given by

$$
\left[\begin{array}{ccc}
0 & \frac{1}{c_{2}} p_{3} & \frac{1}{c_{2}} p_{2} \\
-\frac{1}{c_{2}} p_{3} & 0 & -\frac{1}{c_{2}} p_{1} \\
\frac{1}{c_{1}} p_{2} & \frac{1}{c_{1}} p_{1} & 0
\end{array}\right] .
$$

(i) Assume $\mu \neq 0$. (The state $e_{1}^{0}=e_{3}^{0}$ is dealt with in (iii)). Let $H_{\chi}=H+\chi(C)$ be an energy-Casimir function, i.e.,

$$
H_{\chi}\left(p_{1}, p_{2}, p_{3}\right)=\frac{1}{2 c_{1}} p_{2}^{2}+\frac{1}{2 c_{2}} p_{3}^{2}+\chi\left(p_{1}^{2}+p_{2}^{2}\right)
$$

where $\chi \in C^{\infty}(\mathbb{R})$. The derivative

$$
\mathrm{d} H_{\chi}=\left[2 p_{1} \dot{\chi}\left(p_{1}^{2}+p_{2}^{2}\right) \frac{1}{c_{1}} p_{2}+2 p_{2} \dot{\chi}\left(p_{1}^{2}+p_{2}^{2}\right) \frac{1}{c_{2}} p_{3}\right]
$$

vanishes at $e_{1}^{\mu}$ if $\dot{\chi}\left(\mu^{2}\right)=0$. Then the Hessian (at $\left.e_{1}^{\mu}\right)$

$$
\mathrm{d}^{2} H_{\chi}(\mu, 0,0)=\operatorname{diag}\left(2 \dot{\chi}\left(\mu^{2}\right)+4 \mu^{2} \ddot{\chi}\left(\mu^{2}\right), \frac{1}{c_{1}}+2 \dot{\chi}\left(\mu^{2}\right), \frac{1}{c_{2}}\right)
$$

is positive definite if $\ddot{\chi}\left(\mu^{2}\right)>0$ (and $\dot{\chi}\left(\mu^{2}\right)=0$ ). The function $\chi(x)=\frac{1}{2} x^{2}-\mu^{2} x$ satisfies these requirements. Hence, by the standard energy-Casimir method, $e_{1}^{\mu}$ is stable. 
(ii) The linearization of the system at $e_{2}^{\nu}$ has eigenvalues $\lambda_{1}=0, \lambda_{2,3}= \pm \frac{\nu}{\sqrt{c_{1} c_{2}}}$. Thus $e_{2}^{\nu}$ is unstable.

(iii) Let $H_{\lambda}=\lambda_{0} H+\lambda_{1} C$. Then $\mathrm{d} H_{\lambda}(0,0, \mu)=\left[\begin{array}{lll}0 & 0 & \frac{\lambda_{0} \mu}{c_{2}}\end{array}\right]$ and $\mathrm{d}^{2} H_{\lambda}(0,0, \mu)=\operatorname{diag}\left(2 \lambda_{1}, \frac{\lambda_{0}}{c_{1}}+2 \lambda_{1}, \frac{\lambda_{0}}{c_{2}}\right)$. Suppose $\mu=0$ and let $\lambda_{0}=\lambda_{1}=1$. Then $\mathrm{d} H_{\lambda}(0,0,0)=0$ and $\mathrm{d}^{2} H_{\lambda}(0,0,0)$ is positive definite. On the other hand, suppose $\mu \neq 0$ and let $\lambda_{0}=0, \lambda_{1}=1$. Then $\mathrm{d} H_{\lambda}(0,0, \mu)=0$ and $\mathrm{d}^{2} H_{\lambda}(0,0, \mu)=\operatorname{diag}(2,2,0)$. Also,

$$
\operatorname{ker} \mathrm{d} H\left(e_{3}^{\mu}\right) \cap \operatorname{ker} \mathrm{d} C\left(e_{3}^{\mu}\right)=\operatorname{span}\{(1,0,0),(0,1,0)\}
$$

and so $\left.\mathrm{d}^{2} H_{\lambda}(0,0, \mu)\right|_{W \times W}=\operatorname{diag}(2,2)$ is positive definite. Hence, by the extended energy-Casimir method, $e_{3}^{\mu}$ is stable.

The equilibrium states for (3.5) are

$$
e_{1}^{\mu}=(\mu, 0,0), \quad e_{2}^{\mu}=\left(c_{1}, \mu, 0\right) \quad \text { and } \quad e_{3}^{\nu}=(0,0, \nu)
$$

where $\mu, \nu \in \mathbb{R}, \nu \neq 0$.

Theorem 4.2. The equilibrium states have the following behaviour:

(i) Each equilibrium state $e_{1}^{\mu}$ is unstable if $\mu \in\left[0, c_{1}\right]$ and stable if $\mu \in(-\infty, 0) \cup\left(c_{1}, \infty\right)$.

(ii) Each equilibrium state $e_{2}^{\mu}$ is unstable.

(iii) Each equilibrium state $e_{3}^{\nu}$ is stable.

Proof. The linearization of the system is given by

$$
\left[\begin{array}{ccc}
0 & \frac{1}{c_{2}} p_{3} & \frac{1}{c_{2}} p_{2} \\
-\frac{1}{c_{2}} p_{3} & 0 & -\frac{1}{c_{2}} p_{1} \\
\frac{1}{c_{1}} p_{2} & \frac{1}{c_{1}} p_{1}-1 & 0
\end{array}\right] .
$$

(i) Assume $\mu \in\left(0, c_{1}\right)$. The linearization of the system (at $\left.e_{1}^{\mu}\right)$ has eigenvalues $\lambda_{1}=0, \lambda_{2,3}= \pm \sqrt{\frac{\left(c_{1}-\mu\right) \mu}{c_{1} c_{2}}}$. Thus $e_{1}^{\mu}$ is unstable. Now, assume $\mu=c_{1}$ or $\mu=0$. Then the linearization of the system (at $e_{1}^{\mu}$ ) has eigenvalues $\lambda_{1,2,3}=0$. Thus, as the geometric multiplicity is strictly less than the algebraic multiplicity, $e_{1}^{\mu}$ is unstable.

Assume $\mu \in(-\infty, 0) \cup\left(c_{1}, \infty\right)$. Let $H_{\chi}=H+\chi(C)$ be an energy-Casimir function, i.e.,

$$
H_{\chi}\left(p_{1}, p_{2}, p_{3}\right)=p_{1}+\frac{1}{2 c_{1}} p_{2}^{2}+\frac{1}{2 c_{2}} p_{3}^{2}+\chi\left(p_{1}^{2}+p_{2}^{2}\right)
$$

where $\chi \in C^{\infty}(\mathbb{R})$. The derivative

$$
\mathrm{d} H_{\chi}=\left[1+2 p_{1} \dot{\chi}\left(p_{1}^{2}+p_{2}^{2}\right) \frac{1}{c_{1}} p_{2}+2 p_{2} \dot{\chi}\left(p_{1}^{2}+p_{2}^{2}\right) \frac{1}{c_{2}} p_{3}\right]
$$

vanishes at $e_{1}^{\mu}$ if $\dot{\chi}\left(\mu^{2}\right)=-\frac{1}{2 \mu}$. Then the Hessian (at $e_{1}^{\mu}$ )

$$
\begin{aligned}
\mathrm{d}^{2} H_{\chi}(\mu, 0,0) & =\operatorname{diag}\left(4 \mu^{2} \ddot{\chi}\left(\mu^{2}\right)+2 \dot{\chi}\left(\mu^{2}\right), \frac{1}{c_{1}}+2 \dot{\chi}\left(\mu^{2}\right), \frac{1}{c_{2}}\right) \\
& =\operatorname{diag}\left(4 \mu^{2} \ddot{\chi}\left(\mu^{2}\right)-\frac{1}{\mu}, \frac{1}{c_{1}}-\frac{1}{\mu}, \frac{1}{c_{2}}\right)
\end{aligned}
$$

is positive definite if $\ddot{\chi}\left(\mu^{2}\right)>\frac{1}{4 \mu^{3}}$. The function $\chi(x)=\left(\frac{1}{8 \mu^{3}}+1\right) x^{2}-\frac{3+8 \mu^{3}}{4 \mu} x$ satisfies these requirements. Hence, by the standard energy-Casimir method, $e_{1}^{\mu}$ is stable.

(ii) Assume $\mu \neq 0$. (The case $\mu=0$ has already been dealt with). The linearization of the system (at $e_{2}^{\mu}$ ) has eigenvalues $\lambda_{1}=0, \lambda_{2,3}= \pm \frac{\mu}{\sqrt{c_{1} c_{2}}}$. Thus $e_{2}^{\mu}$ is unstable. 


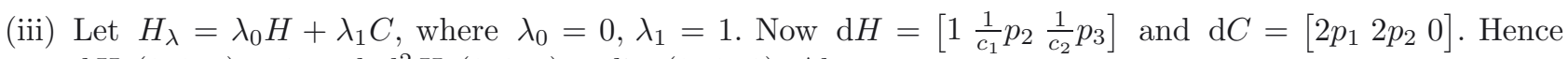
$\mathrm{d} H_{\lambda}(0,0, \nu)=0$ and $\mathrm{d}^{2} H_{\lambda}(0,0, \nu)=\operatorname{diag}(2,2,0)$. Also,

$$
\operatorname{ker} \mathrm{d} H\left(e_{3}^{\nu}\right) \cap \operatorname{ker} \mathrm{d} C\left(e_{3}^{\nu}\right)=\operatorname{span}\left\{\left(-\nu, 0, c_{2}\right),(0,1,0)\right\}
$$

and so $\left.\mathrm{d}^{2} H_{\lambda}(0,0, \nu)\right|_{W \times W}=\operatorname{diag}\left(2 \nu^{2}, 2\right)$ is positive definite. Hence, by the extended energy-Casimir method, $e_{3}^{\nu}$ is stable.

The equilibrium states for (3.6) are

$$
e^{\mu}=(0,0, \mu), \quad \mu \in \mathbb{R} .
$$

Theorem 4.3. Each equilibrium state $e^{\mu}$ is stable.

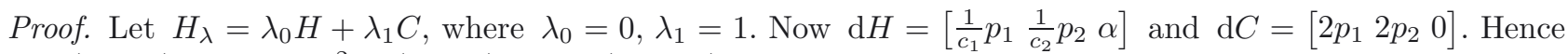
$\mathrm{d} H_{\lambda}(0,0, \mu)=0$ and $\mathrm{d}^{2} H_{\lambda}(0,0, \mu)=\operatorname{diag}(2,2,0)$. Also,

$$
\operatorname{ker} \mathrm{d} H\left(e^{\mu}\right) \cap \operatorname{ker} \mathrm{d} C\left(e^{\mu}\right)=\operatorname{span}\{(1,0,0),(0,1,0)\}
$$

and so $\left.\mathrm{d}^{2} H_{\lambda}(0,0, \mu)\right|_{W \times W}=\operatorname{diag}(2,2)$ is positive definite. Hence, by the extended energy-Casimir method, $e^{\mu}$ is stable.

\subsection{Integral curves}

We give qualitative descriptions of the integral curves of $\vec{H}_{1}, \vec{H}_{2}$, and $\vec{H}_{3}$. Let $\mathcal{E}_{1}, \mathcal{E}_{2}$, and $\mathcal{E}_{3}$ denote the set of equilibrium points for $\vec{H}_{1}, \vec{H}_{2}$, and $\vec{H}_{3}$, respectively.

Proposition 4.4. The level sets

$$
\mathcal{C}_{i}=\left(C^{-1}\left(c_{0}\right) \cap H_{i}^{-1}\left(h_{i}\right)\right) \backslash \mathcal{E}_{i}, \quad i=1,2,3
$$

are bounded embedded 1-submanifolds of $\mathfrak{s e}(2)^{*}$ for $c_{0}>0, h_{1}>0, h_{2}>-\sqrt{c_{0}}$, and $h_{3} \in \mathbb{R}$.

(Some typical cases for these sets are graphed in Figs. 1-3).

Proof. Let $F_{1}: \mathfrak{s e}(2)^{*} \backslash \mathcal{E}_{1} \rightarrow \mathbb{R}^{2}, p \mapsto\left(C(p), H_{1}(p)\right)$. Note that, as $\mathcal{E}_{1}$ is closed, $\mathfrak{s e}(2)^{*} \backslash \mathcal{E}_{1}$ is open and thus an embedded 3 -submanifold of $\mathfrak{s e}(2)^{*}$. We have

$$
D F_{1}(p)=\left[\begin{array}{ccc}
2 p_{1} & 2 p_{2} & 0 \\
0 & \frac{1}{c_{1}} p_{2} & \frac{1}{c_{2}} p_{3}
\end{array}\right]
$$

which has full rank unless $p_{1}=0$ and $p_{3}=0$. However, $\left(0, p_{2}, 0\right) \in \mathcal{E}_{1}$. Thus $\mathcal{C}_{1}=F^{-1}\left(c_{0}, h_{1}\right)$ is an embedded 1 -submanifold of $\mathfrak{s e}(2)^{*}$. For $p=\left(p_{1}, p_{2}, p_{3}\right) \in \mathcal{C}_{1}$, we have $p_{1}^{2}+p_{2}^{2}=c_{0}$ and $\frac{1}{2}\left(\frac{1}{c_{1}} p_{2}^{2}+\frac{1}{c_{2}} p_{3}^{2}\right)=h_{1}$. Hence $p_{1}^{2} \leq c_{0}, p_{2}^{2} \leq c_{0}$, and $p_{3}^{2} \leq 2 h_{1} c_{2}$. Thus $\mathcal{C}_{1}$ is bounded.

A similar argument shows that $\mathcal{C}_{2}$ and $\mathcal{C}_{3}$ are bounded embedded 1-submanifolds of $\mathfrak{s e}(2)^{*}$. The conditions on $c_{0}, h_{1}$, and $h_{2}$ are required such that the sets $\mathcal{C}_{1}, \mathcal{C}_{2}$, and $\mathcal{C}_{3}$ are nonempty.

As $H_{i}$ and $C$ are constants of the motion, any non-constant integral curve $p(\cdot)$ of $\vec{H}_{i}$ evolves on $\mathcal{C}_{i}$ (where $c_{0}=C(p(0))$ and $\left.h_{i}=H_{i}(p(0))\right)$. Moreover, as each $\mathcal{C}_{i}$ is bounded, any integral curve lies in a compact subset of $\mathfrak{s e}(2)^{*}$. Hence (see, e.g., [1]) 
Corollary 4.5. The vector fields $\vec{H}_{1}, \vec{H}_{2}$ and $\vec{H}_{3}$ are complete.

We now describe the integral curves of $\vec{H}_{1}, \vec{H}_{2}$, and $\vec{H}_{3}$. Let $p(\cdot)$ be a maximal integral curve of a complete vector field on $\mathfrak{s e}(2)^{*}$. A point $p \in \mathfrak{s e}(2)^{*}$ is an $\omega$-limit point of $p(\cdot)$ if there exists a sequence $\left(t_{n}\right)$ such that $t_{n} \rightarrow \infty$ and $p\left(t_{n}\right) \rightarrow p$. Similarly, if there exists a sequence $\left(t_{n}\right)$ such that $t_{n} \rightarrow-\infty$ and $p\left(t_{n}\right) \rightarrow q$, then $q$ is an $\alpha$-limit point. The set of all $\alpha$-limit points of $p(\cdot)$ is denoted $\lim _{\alpha} p(\cdot)$; the set of all $\omega$-limit points of $p(\cdot)$ is denoted $\lim _{\omega} p(\cdot)$.

Theorem 4.6. Let $p(\cdot): \mathbb{R} \rightarrow \mathfrak{s e}(2)^{*}$ be a non-constant maximal integral curve of $\vec{H}_{i}$. Let $\mathcal{C}_{i}^{0}$ be the corresponding connected component of $\mathcal{C}_{i}$ containing $p(0)$.

1. Suppose $p(\cdot)$ is an integral curve of $\vec{H}_{1}$.

(a) If $\mathcal{C}_{1}^{0}$ is closed (or equivalently $\mathrm{cl}_{1}^{0} \cap \mathcal{E}_{1}=\emptyset$ ), then $p(\cdot)$ is periodic.

(b) If $\mathcal{C}_{1}^{0}$ is not closed (or equivalently $\operatorname{cl} \mathcal{C}_{1}^{0} \cap \mathcal{E}_{1} \neq \emptyset$ ), then $p(\cdot)$ is bounded and the limit sets $\lim _{\omega} p(\cdot$ ) and $\lim _{\alpha} p(\cdot)$ are singletons in $\mathcal{E}_{1}$.

In either case, $p(\cdot)$ has image $\mathcal{C}_{1}^{0}$.

2. Suppose $p(\cdot)$ is an integral curve of $\vec{H}_{2}$.

(a) If $\mathcal{C}_{2}^{0}$ is closed (or equivalently $\mathrm{cl} \mathcal{C}_{2}^{0} \cap \mathcal{E}_{2}=\emptyset$ ), then $p(\cdot)$ is periodic.

(b) If $\mathcal{C}_{2}^{0}$ is not closed (or equivalently $\operatorname{cl} \mathcal{C}_{2}^{0} \cap \mathcal{E}_{2} \neq \emptyset$ ), then $p(\cdot)$ is bounded and the limit sets $\lim _{\omega} p(\cdot$ ) and $\lim _{\alpha} p(\cdot)$ are singletons in $\mathcal{E}_{2}$.

In either case, $p(\cdot)$ has image $\mathcal{C}_{2}^{0}$.

3. If $p(\cdot)$ is an integral curve of $\vec{H}_{3}$, then $p(\cdot)$ is periodic and has image $\mathcal{C}_{3}^{0}$.

Proof. First we show that $\mathcal{C}_{i}^{0}$ is closed if and only if $\operatorname{cl}_{i}^{0} \cap \mathcal{E}_{i}=\emptyset$ (for $i=1,2,3$ ). If $\mathcal{C}_{i}^{0}$ is closed, then $\operatorname{cl} \mathcal{C}_{i}^{0}=\mathcal{C}_{i}^{0} \subseteq\left(C^{-1}\left(c_{0}\right) \cap H_{i}^{-1}\left(h_{i}\right)\right) \backslash \mathcal{E}_{i}$ and so $\operatorname{cl} \mathcal{C}_{i}^{0} \cap \mathcal{E}_{i}=\emptyset$. Conversely, suppose $\operatorname{cl} \mathcal{C}_{i}^{0} \cap \mathcal{E}_{i}=\emptyset$. Then $\operatorname{cl} \mathcal{C}_{i}^{0} \subset$ $\mathfrak{s e}(2)^{*} \backslash \mathcal{E}_{i}$ and $\operatorname{cl} \mathcal{C}_{i}^{0} \subseteq C^{-1}\left(c_{0}\right) \cap H_{i}^{-1}\left(h_{i}\right)$, i.e., cl $\mathcal{C}_{i}^{0} \subseteq \mathcal{C}_{i}$. However, as $\mathcal{C}_{i}^{0}$ is connected, cl $\mathcal{C}_{i}^{0}$ is connected. Thus $\operatorname{cl} \mathcal{C}_{i}^{0}=\mathcal{C}_{i}^{0}$.

(1) We have that $p(\cdot)$ evolves on $\mathcal{C}_{1}^{0}$. As $\mathcal{C}_{1}^{0}$ is a connected 1-manifold, it is diffeomorphic to either the circle $\mathbb{S}$ or the real line $\mathbb{R}$. (1a) Suppose $\mathcal{C}_{1}^{0}$ is closed. Then $\mathcal{C}_{1}^{0}$ is diffeomorphic to $\mathbb{S}$. Let $X$ be the push-forward (to $\mathbb{S}$ ) of the restriction of $\vec{H}_{1}$ to $\mathcal{C}_{1}^{0} . X$ is complete and nonzero everywhere. As $\mathbb{S}$ is compact, $\|X\|$ attains a positive minimum. Hence there is a finite interval $\left[t_{0}, t_{1}\right]$ in which any integral curve of $X$ covers $\mathbb{S}$. Hence any maximal integral curve of $X$ is periodic. Consequently, $p(\cdot)$ is periodic (as it is diffeomorphic to a periodic integral curve) and has image $\mathcal{C}_{1}^{0}$.

(1b) Suppose $\mathcal{C}_{1}^{0}$ is not closed. As $\mathcal{C}_{1}$ is bounded, the $\operatorname{limit}$ sets $\lim _{\alpha} p(\cdot)$ and $\lim _{\omega} p(\cdot)$ are non-empty, connected, compact subsets of $\mathfrak{s e}(2)^{*}\left(c f\right.$. [20]). Also $p(\cdot)$ is bounded. Now $\mathcal{C}_{1}^{0}$ is diffeomorphic to $\mathbb{R}$. Let $X$ be the push-forward (to $\mathbb{R}$ ) of the restriction of $\vec{H}_{1}$ to $\mathcal{C}_{1}^{0} . X$ is complete and nonzero everywhere. Hence, we may assume $X(q)>0$ for $q \in \mathbb{R}$. Let $q(\cdot): \mathbb{R} \rightarrow \mathbb{R}$ be a maximal integral curve of $X$. For every compact interval $\left[q\left(t_{0}\right), q_{1}\right]$ or $\left[q_{0}, q\left(t_{1}\right)\right]$ in $\mathbb{R},\|X\|$ attains a positive minimum. Hence (for every such interval) there exists a $T \geq 0$ such that $q\left(t_{0}+T\right)>q_{1}$ or $q\left(t_{1}-T\right)<q_{0}$, respectively. From this we draw two conclusions. First, any maximal integral curve of $X$ covers $\mathbb{R}$. Second, if $\left(t_{n}\right)$ is a sequence in $\mathbb{R}$ such that $t_{n} \rightarrow \pm \infty$ and $q(\cdot): \mathbb{R} \rightarrow \mathbb{R}$ is a maximal integral curve, then $\left(q\left(t_{n}\right)\right)$ does not converge in $\mathbb{R}$. Consequently, the image of $p(\cdot)$ is $\mathcal{C}_{1}^{0}$. Also, there are no $\alpha$ - or $\omega$-limit points of $p(\cdot)$ in $\mathcal{C}_{1}^{0}$. However, any $\alpha$ - or $\omega$-limit point must be in $\operatorname{cl}_{1}^{0} \subseteq C^{-1}\left(c_{0}\right) \cap H_{1}^{-1}\left(h_{1}\right)$.

We claim that $\lim _{\alpha} p(\cdot) \cap \mathcal{C}_{1}=\emptyset$ and $\lim _{\omega} p(\cdot) \cap \mathcal{C}_{1}=\emptyset$. Suppose there exists $p \in \lim _{\alpha} p(\cdot) \cap \mathcal{C}_{1}$ or $p \in \lim _{\omega} p(\cdot) \cap \mathcal{C}_{1}$. Then $p \in \operatorname{cl}_{1}^{0}, p \in \mathcal{C}_{1}$, and $p \notin \mathcal{C}_{1}^{0}$. Hence $\mathcal{C}_{1}^{0} \cup\{p\}$ is connected and $\mathcal{C}_{1}^{0} \cup\{p\} \subseteq \mathcal{C}_{1}$. Thus $\mathcal{C}_{1}^{0} \cup\{p\}$ is contained in the connected component $\mathcal{C}_{1}^{0}$ of $\mathcal{C}_{1}$ containing $p(0)$, a contradiction. So we have

$$
\lim _{\alpha} p(\cdot) \subseteq C^{-1}\left(c_{0}\right) \cap H_{1}^{-1}\left(h_{1}\right) \quad \lim _{\alpha} p(\cdot) \cap\left(\left(C^{-1}\left(c_{0}\right) \cap H_{1}^{-1}\left(h_{1}\right)\right) \backslash \mathcal{E}_{1}\right)=\emptyset
$$

and similarly for $\lim _{\omega} p(\cdot)$. Hence

$$
\lim _{\alpha} p(\cdot), \lim _{\omega} p(\cdot) \subseteq C^{-1}\left(c_{0}\right) \cap H_{1}^{-1}\left(h_{1}\right) \cap \mathcal{E}_{1} .
$$


A simple calculation shows that $C^{-1}\left(c_{0}\right) \cap H_{1}^{-1}\left(h_{1}\right) \cap \mathcal{E}_{1}$ is finite and hence completely disconnected. Therefore, as $\lim _{\alpha} p(\cdot)$ and $\lim _{\omega} p(\cdot)$ are non-empty and connected, $\lim _{\alpha} p(\cdot)$ and $\lim _{\omega} p(\cdot)$ are singletons.

For (2), a similar argument yields the result. (3) We show that $\mathcal{C}_{3}^{0}$ is closed by showing that $\operatorname{cl} \mathcal{C}_{3}^{0} \cap \mathcal{E}_{3}=\emptyset$. The result then follows in the same way as for (1a). Suppose there exists $p \in \operatorname{cl} \mathcal{C}_{3}^{0} \cap \mathcal{E}_{3}$. Then $p \in C^{-1}\left(c_{0}\right) \cap$ $H_{3}^{-1}\left(h_{3}\right) \cap \mathcal{E}_{3}$. Hence, as $p \in \mathcal{E}_{3}, p=(0,0, \mu)$ for some $\mu \in \mathbb{R}$. Hence, as $p \in C^{-1}\left(c_{0}\right)$, we get $c_{0}=0$, a contradiction.

\section{ExpliCit INTEGRATION}

The reduced Hamilton equations (3.4)-(3.6) can be integrated by Jacobi elliptic functions. (In fact, (3.6) can easily be integrated by trigonometric functions). In each of these cases, we obtain explicit expressions for the integral curves of $\vec{H}$. Before producing these results, we outline the basic approach employed in obtaining them. (In each case, MAthematica is utilised to facilitate calculations). A similar approach was used in [2] for single-input systems on SE (2).

Note 5.1. In this section we consider only non-constant integral curves.

First, we fix a Hamiltonian vector field $\vec{H}$ (specifically (3.4) or (3.5)). We then partition the set of all initial conditions; this enables one to produce a single explicit expression for each (sub)case. The first separation is made by considering when the level surfaces, defined by the constants of motion $C$ and $H$, are tangent to one another. This level of separation is sufficient for solving (3.4), but further partitioning (made retrospectively) is needed in solving (3.5).

Next, we suppose that $\bar{p}(\cdot):(-\varepsilon, \varepsilon) \rightarrow \mathfrak{s e}(2)^{*}$ is an integral curve of $\vec{H}$ (satisfying some appropriate conditions). We let $h_{0}=H(\bar{p}(0))$ and $c_{0}=C(\bar{p}(0))>0$. Then, as $\bar{p}(\cdot)$ solves (3.4) or (3.5), we get that

$$
\begin{aligned}
\frac{\mathrm{d}}{\mathrm{d} t} \bar{p}_{2} & = \pm \sqrt{\frac{1}{c_{1} c_{2}}\left(c_{0}-\bar{p}_{2}^{2}\right)\left(2 h_{0} c_{1}-\bar{p}_{2}^{2}\right)} \\
\frac{\mathrm{d}}{\mathrm{d} t} \bar{p}_{1} & = \pm \sqrt{\frac{1}{c_{1} c_{2}}\left(c_{0}-\bar{p}_{1}^{2}\right)\left(2 c_{1} h_{0}-c_{0}-2 c_{1} \bar{p}_{1}+\bar{p}_{1}^{2}\right)}
\end{aligned}
$$

respectively. In most cases, the respective (separable) differential equation is transformed into standard form (see [4] or [15]). A formula for an elliptic integral is then applied to obtain an expression for $\bar{p}_{2}(t)$ or $\bar{p}_{1}(t)$, respectively. (Observe however that (5.1) is already in standard form). Often, a good deal of further simplification is then performed. Next, by use of the constants of motion $C$ and $H$, expressions for $\bar{p}_{1}(t), \bar{p}_{2}(t)$ and $\bar{p}_{3}(t)$ are determined up to a choice of sign and organised so as to be smooth (again involving further simplification).

Accordingly, we get a prospective (smooth) integral curve $\bar{p}(\cdot)$ whose domain is extended to $\mathbb{R}$. In some special cases, the prospective integral curve $\bar{p}(\cdot)$ may be produced by a limiting process from other results already obtained, or by directly solving the differential equation, as is the case with (3.6). Then, by explicitly differentiating $\bar{p}(\cdot)$, we verify for which choices of sign $\bar{p}(\cdot)$ is an integral curve of $\vec{H}$. (This is then further verified by solving the respective differential equation numerically for some suitable initial condition). Finally, we show that any other integral curve $p(\cdot):(-\varepsilon, \varepsilon) \rightarrow \mathfrak{s e}(2)^{*}$ of $\vec{H}$, evolving on $C^{-1}\left(c_{0}\right) \cap H^{-1}\left(h_{0}\right)$, is identical to $\bar{p}(\cdot)$ up to a translation in the independent variable and an allowable choice of sign.

Various properties of the Jacobi elliptic functions are involved in making the above mentioned calculations $(c f .[4,15])$. In particular, we use the periodicity properties $(e . g ., \operatorname{sn}(x+K, k)=\operatorname{cd}(x, k))$, relations of squares (e.g., $\left.1-k^{2} \operatorname{sn}^{2}(x, k)=\operatorname{dn}^{2}(x, k)\right)$ and half-angle formulas $\left(e . g ., \operatorname{cn}^{2}\left(\frac{1}{2} x, k\right)=\frac{\operatorname{cn}(x, k)+\operatorname{dn}(x, k)}{1+\operatorname{dn}(x, k)}\right)$.

We now produce the results for each typical case. (A summary of these results may be found in the appendix). Only for Theorem 5.10 will a proof detailing the method used to obtain the result be provided. For the remaining results we omit details pertaining to finding a maximal integral curve (they follow the same approach or are easy to obtain by straightforward integration) and only verify that every other integral curve is identical up to a translation in the independent variable. 


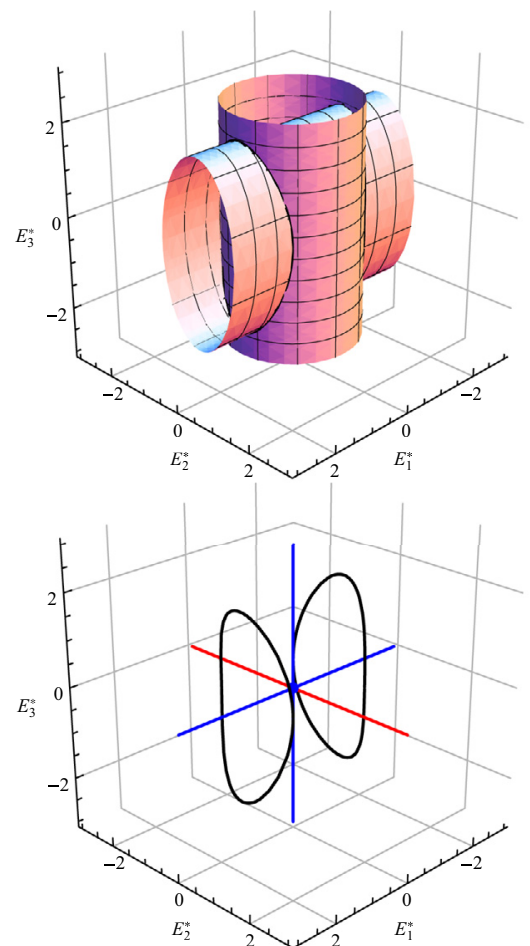

(A) $c_{0}>2 c_{1} h_{0}$
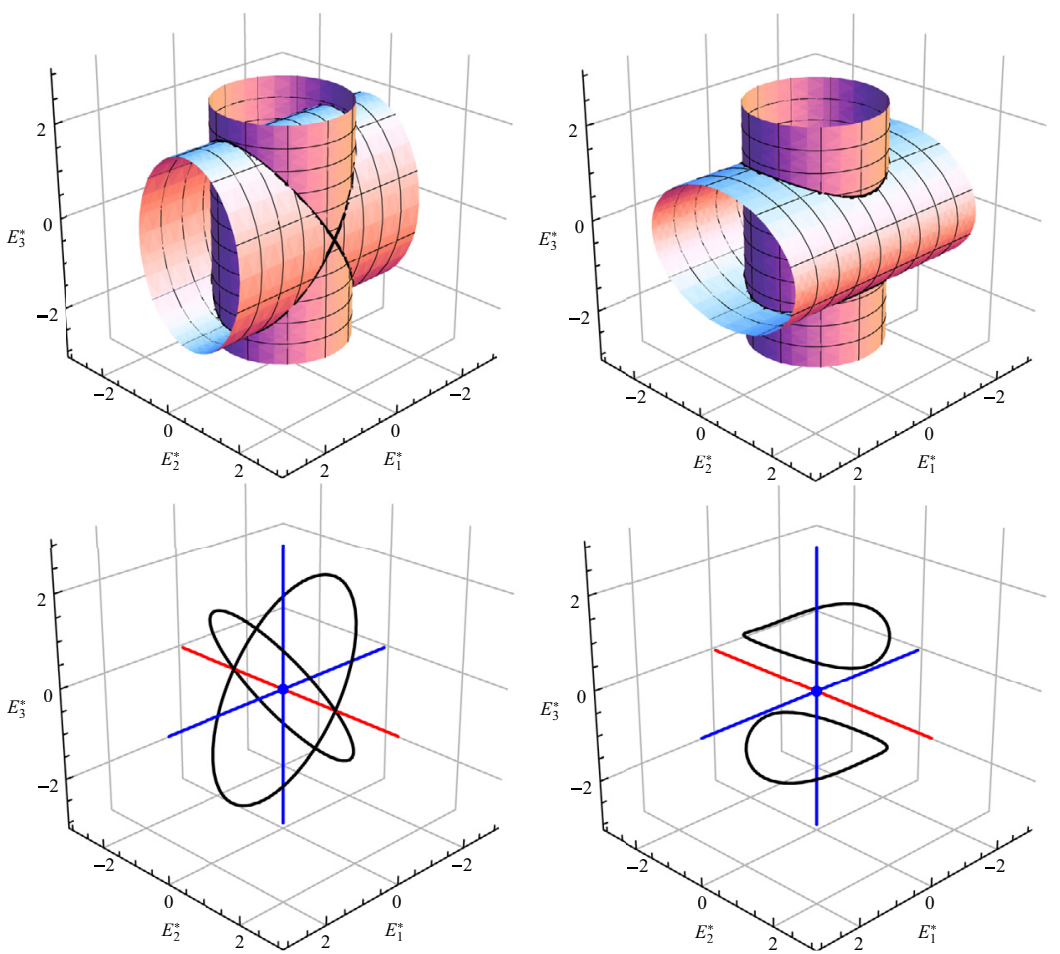

(в) $c_{0}=2 c_{1} h_{0}$

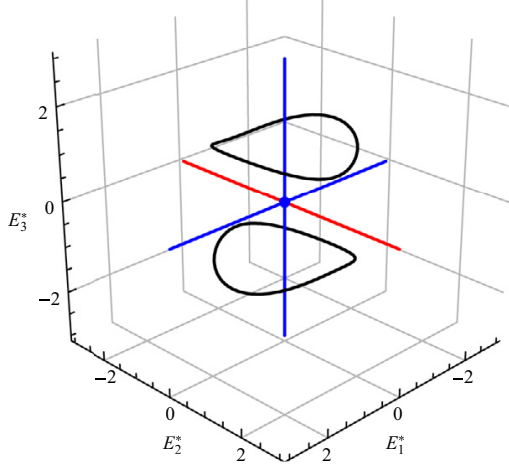

(C) $c_{0}<2 c_{1} h_{0}$

Figure 1. Typical cases of LiCP(1).

\subsection{Homogeneous systems}

There are three typical cases for the reduced extremal equations (3.4), corresponding to (a) $c_{0}>2 c_{1} h_{0}$, (b) $c_{0}=2 c_{1} h_{0}$ and (c) $c_{0}<2 c_{1} h_{0}$. In Figure 1, we graph the level sets of $H$ and $C$ and their intersection for some suitable values of $h_{0}, c_{0}, c_{1}$ and $c_{2}$. The stable equilibrium points (illustrated in blue) and unstable equilibrium points (illustrated in red), as presented in Theorem 4.1, are also plotted in each case.

We begin our presentation of the integral curves of (3.4) with case (a). (In the following theorem, formula (2.7) is used to obtain a prospective integral curve).

Theorem 5.2 (case a). Suppose $p(\cdot):(-\varepsilon, \varepsilon) \rightarrow \mathfrak{s e}(2)^{*}$ is an integral curve of $\vec{H}$ such that $H(p(0))=h_{0}>0$, $C(p(0))=c_{0}>0$ and $c_{0}>2 c_{1} h_{0}$. Then there exists $t_{0} \in \mathbb{R}$ and $\sigma \in\{-1,1\}$ such that $p(t)=\bar{p}\left(t+t_{0}\right)$ for $t \in(-\varepsilon, \varepsilon)$, where

$$
\left\{\begin{array}{l}
\bar{p}_{1}(t)=\sigma \sqrt{c_{0}} \operatorname{dn}(\Omega t, k) \\
\bar{p}_{2}(t)=\sqrt{2 c_{1} h_{0}} \operatorname{sn}(\Omega t, k) \\
\bar{p}_{3}(t)=-\sigma \sqrt{2 c_{2} h_{0}} \operatorname{cn}(\Omega t, k) .
\end{array}\right.
$$

Here $k=\sqrt{\frac{2 c_{1} h_{0}}{c_{0}}}$ and $\Omega=\sqrt{\frac{c_{0}}{c_{1} c_{2}}}$.

Proof. Verification that $\bar{p}(\cdot)$ is an integral curve satisfying $H(\bar{p}(0))=h_{0}, C(p(0))=c_{0}$ whenever $c_{0}>2 c_{1} h_{0}>$ 0 is straightforward. We have $p_{1}(0)^{2}+p_{2}(0)^{2}=c_{0}$ and $\frac{1}{c_{1}} p_{2}(0)^{2}+\frac{1}{c_{2}} p_{3}(0)^{2}=2 h_{0}$. Thus $p_{2}(0)^{2} \leq 2 h_{0} c_{1}$ and so 
$p_{1}(0)^{2}=c_{0}-p_{2}(0)^{2} \geq c_{0}-2 h_{0} c_{1}>0$. Let $\sigma=\operatorname{sgn}\left(p_{1}(0)\right)$ (we have $\sigma \neq 0$ ). Furthermore, $-\sqrt{2 h_{0} c_{2}} \leq p_{3}(0) \leq$ $\sqrt{2 h_{0} c_{2}}, \bar{p}_{3}(0)=-\sigma \sqrt{2 c_{2} h_{0}}$ and $\bar{p}_{3}\left(\frac{2 K}{\Omega}\right)=\sigma \sqrt{2 c_{2} h_{0}}$. Hence there exists $t_{1} \in \mathbb{R}$ such that $\bar{p}_{3}\left(t_{1}\right)=p_{3}(0)$. Now

$$
p_{2}(0)^{2}=2 h_{0} c_{1}-\frac{c_{1}}{c_{2}} p_{3}(0)^{2}=2 h_{0} c_{1}-\frac{c_{1}}{c_{2}} \bar{p}_{3}\left(t_{1}\right)^{2}=\bar{p}_{2}\left(t_{1}\right)^{2} .
$$

Thus $p_{2}(0)= \pm \bar{p}_{2}\left(t_{1}\right)$. However, $\bar{p}_{2}(-t)=-\bar{p}_{2}(t)$ and $\bar{p}_{3}(-t)=\bar{p}_{3}(t)$. Therefore there exists $t_{0} \in \mathbb{R}$ (either $t_{0}=t_{1}$ or $\left.t_{0}=-t_{1}\right)$ such that $p_{3}(0)=\bar{p}_{3}\left(t_{0}\right)$ and $p_{2}(0)=\bar{p}_{2}\left(t_{0}\right)$. Also

$$
p_{1}(0)^{2}=c_{0}-p_{2}(0)^{2}=c_{0}-\bar{p}_{2}\left(t_{0}\right)^{2}=\bar{p}_{1}\left(t_{0}\right)^{2} .
$$

Hence, as $\operatorname{sgn}\left(p_{1}(0)\right)=\sigma=\operatorname{sgn}\left(\bar{p}_{1}\left(t_{0}\right)\right)$, we get $p_{1}(0)=\bar{p}_{1}\left(t_{0}\right)$. Thus $p(0)=\bar{p}\left(t_{0}\right)$. Consequently the integral curves $t \mapsto p(t)$ and $t \mapsto \bar{p}\left(t+t_{0}\right)$ solve the same Cauchy problem, and therefore are identical.

Next, by limiting $c_{0} \rightarrow 2 c_{1} h_{0}$ in Theorem 5.2 (and allowing for possible changes in sign), we get a prospective integral curve for case (b).

Proposition 5.3 (case b). Suppose $p(\cdot):(-\varepsilon, \varepsilon) \rightarrow \mathfrak{s e}(2)^{*}$ is an integral curve of $\vec{H}$ such that $H(p(0))=h_{0}>$ $0, C(p(0))=c_{0}>0$ and $c_{0}=2 c_{1} h_{0}$. Then there exists $t_{0} \in \mathbb{R}$ and $\sigma_{1}, \sigma_{2} \in\{-1,1\}$ such that $p(t)=\bar{p}\left(t+t_{0}\right)$ for $t \in(-\varepsilon, \varepsilon)$, where

$$
\left\{\begin{array}{l}
\bar{p}_{1}(t)=\sigma_{1} \sigma_{2} \sqrt{c_{0}} \operatorname{sech}(\Omega t) \\
\bar{p}_{2}(t)=\sigma_{1} \sqrt{c_{0}} \tanh (\Omega t) \\
\bar{p}_{3}(t)=-\sigma_{2} \sqrt{\frac{c_{0} c_{2}}{c_{1}}} \operatorname{sech}(\Omega t) .
\end{array}\right.
$$

Here $\Omega=\sqrt{\frac{c_{0}}{c_{1} c_{2}}}$.

Proof. Let $\sigma_{2}=-\operatorname{sgn}\left(p_{3}(0)\right)$ and let $\sigma_{1}=\sigma_{2} \operatorname{sgn}\left(p_{1}(0)\right)$. We have $p_{1}(0)^{2}+p_{2}(0)^{2}=c_{0}$. Thus $-\sqrt{c_{0}} \leq p_{2}(0) \leq$ $\sqrt{c_{0}}$ and so there exists $t_{0} \in \mathbb{R}$ such that $p_{2}(0)=\bar{p}_{2}\left(t_{0}\right)$. A simple computation then yields $p(0)=\bar{p}\left(t_{0}\right)$. (It is also simple to verify that $\sigma_{1} \neq 0$ and $\sigma_{2} \neq 0$ provided that $p(\cdot)$ is not constant). Consequently the integral curves $t \mapsto p(t)$ and $t \mapsto \bar{p}\left(t+t_{0}\right)$ solve the same Cauchy problem, and therefore are identical.

Lastly, for case (c), we obtain a prospective integral curve by use of formula (2.7).

Theorem 5.4 (case c). Suppose $p(\cdot):(-\varepsilon, \varepsilon) \rightarrow \mathfrak{s e}(2)^{*}$ is an integral curve of $\vec{H}$ such that $H(p(0))=h_{0}>0$, $C(p(0))=c_{0}>0$ and $c_{0}<2 c_{1} h_{0}$. Then there exists $t_{0} \in \mathbb{R}$ and $\sigma \in\{-1,1\}$ such that $p(t)=\bar{p}\left(t+t_{0}\right)$ for $t \in(-\varepsilon, \varepsilon)$, where

$$
\left\{\begin{array}{l}
\bar{p}_{1}(t)=\sigma \sqrt{c_{0}} \operatorname{cn}(\Omega t, k) \\
\bar{p}_{2}(t)=\sqrt{c_{0}} \operatorname{sn}(\Omega t, k) \\
\bar{p}_{3}(t)=-\sigma \sqrt{2 c_{2} h_{0}} \operatorname{dn}(\Omega t, k) .
\end{array}\right.
$$

Here $k=\sqrt{\frac{c_{0}}{2 c_{1} h_{0}}}$ and $\Omega=\sqrt{\frac{2 h_{0}}{c_{2}}}$.

Proof. Let $\sigma=-\operatorname{sgn}\left(p_{3}(0)\right)$. We have $p_{1}(0)^{2}+p_{2}(0)^{2}=c_{0}$ and $\frac{1}{c_{1}} p_{2}(0)^{2}+\frac{1}{c_{2}} p_{3}(0)^{2}=2 h_{0}$. Hence $p_{2}(0)^{2} \leq c_{0}$ and so $p_{3}(0)^{2} \geq \frac{c_{2}}{c_{1}}\left(2 c_{1} h_{0}-c_{0}\right)>0$. Thus $\sigma \neq 0$. Also, $-\sqrt{c_{0}} \leq p_{2}(0) \leq \sqrt{c_{0}}, \bar{p}_{2}\left(\frac{K}{\Omega}\right)=\sqrt{c_{0}}$ and $\bar{p}_{2}\left(\frac{3 K}{\Omega}\right)=$ $-\sqrt{c_{0}}$. Therefore there exists $t_{1} \in\left[\frac{K}{\Omega}, \frac{3 K}{\Omega}\right]$ such that $p_{2}(0)=\bar{p}_{2}\left(t_{1}\right)$. We have

$$
p_{1}(0)^{2}=c_{0}-p_{2}(0)^{2}=c_{0}-\bar{p}_{2}\left(t_{1}\right)^{2}=\bar{p}_{1}\left(t_{1}\right)^{2} .
$$


Thus $p_{1}(0)= \pm \bar{p}_{1}\left(t_{1}\right)$. Now $\bar{p}_{1}\left(-t+\frac{2 K}{\Omega}\right)=-\bar{p}_{1}(t)$ and $\bar{p}_{2}\left(-t+\frac{2 K}{\Omega}\right)=\bar{p}_{2}(t)$. Hence there exists $t_{0} \in \mathbb{R}\left(t_{0}=t_{1}\right.$ or $\left.t_{1}=-t_{1}+\frac{2 K}{\Omega}\right)$ such that $p_{1}(0)=\bar{p}_{1}\left(t_{0}\right)$ and $p_{2}(0)=\bar{p}_{2}\left(t_{0}\right)$. Next

$$
p_{3}(0)^{2}=2 h_{0} c_{2}-\frac{c_{2}}{c_{1}} p_{2}(0)^{2}=2 h_{0} c_{2}-\frac{c_{2}}{c_{1}} \bar{p}_{2}\left(t_{0}\right)^{2}=\bar{p}_{3}\left(t_{0}\right)^{2} .
$$

Hence, as $\operatorname{sgn}\left(p_{3}(0)\right)=\sigma=\operatorname{sgn}\left(\bar{p}_{3}\left(t_{0}\right)\right), p_{3}(0)=\bar{p}_{3}\left(t_{0}\right)$. Thus $p(0)=\bar{p}\left(t_{0}\right)$. Consequently the integral curves $t \mapsto p(t)$ and $t \mapsto \bar{p}\left(t+t_{0}\right)$ solve the same Cauchy problem, and therefore are identical.

Remark 5.5. These three results are similar to those found by Sachkov [26]. However, our approach and formulation are different. The control problem LiCP(1), or rather, the associated sub-Riemannian problem, is further studied in $[17,27,28]$.

\subsection{Inhomogeneous systems}

In order to separate qualitatively different cases of (3.5), we determine at which points (and for what values of $h_{0}, c_{0}, c_{1}$ and $\left.c_{2}\right)$ the cylinder $C^{-1}\left(c_{0}\right)$ and the paraboloid $H^{-1}\left(h_{0}\right)$ are tangent to one another. If they are tangent at a point $p=\left(p_{1}, p_{2}, p_{3}\right)$, then the gradients of the functions defining these level surfaces at $p$ must be parallel, i.e.,

$$
\nabla C(p)=\left[\begin{array}{lll}
2 p_{1} & 2 p_{2} & 0
\end{array}\right]=r\left[1 \frac{1}{c_{1}} p_{2} \frac{1}{c_{2}} p_{3}\right]=r \nabla H(p)
$$

for some $r \in \mathbb{R}$. There are three distinct possibilities:

$$
\left\{\begin{array} { l } 
{ p _ { 1 } = 0 } \\
{ p _ { 2 } = 0 } \\
{ p _ { 3 } = \pm \sqrt { 2 h _ { 0 } c _ { 2 } } }
\end{array} \quad \left\{\begin{array} { l } 
{ p _ { 1 } = c _ { 1 } } \\
{ p _ { 2 } = \pm \sqrt { c _ { 0 } - c _ { 1 } ^ { 2 } } \neq 0 } \\
{ p _ { 3 } = 0 }
\end{array} \quad \left\{\begin{array}{l}
p_{1} \neq 0 \\
p_{2}=0 \\
p_{3}=0
\end{array}\right.\right.\right.
$$

The first case corresponds to a constant solution and is therefore ignored. By back substitution (into $C$ and $H$ ), the third case yields $h_{0}^{2}=c_{0}$; this motivates us to distinguish between the three signs for $h_{0}^{2}-c_{0}$. (Observe however that the cases $h_{0}=-\sqrt{c_{0}}$ and $c_{0}=0$ correspond to constant solutions, whereas the situation $h_{0}<-\sqrt{c_{0}}$ is impossible). The second case only occurs when $c_{0}-c_{1}^{2}>0$ (and is hence distinguished from the case $\left.c_{0}-c_{1}^{2} \leq 0\right)$. Back substitution yields $h_{0}=\frac{1}{2 c_{1}}\left(c_{0}+c_{1}^{2}\right)$, motivating another separation of three cases. However, not all combinations of these cases are possible.

Lemma 5.6. If $c_{0}-c_{1}^{2}>0$ and $h_{0} \geq \frac{1}{2 c_{1}}\left(c_{0}+c_{1}^{2}\right)$, then $h_{0}>\sqrt{c_{0}}$.

Proof. It suffices to show that $\frac{1}{2 c_{1}}\left(c_{0}+c_{1}^{2}\right)>\sqrt{c_{0}}$. Now

$$
\begin{array}{rlrlrl} 
& & & \frac{1}{2 c_{1}}\left(c_{0}+c_{1}^{2}\right) & >\sqrt{c_{0}} \\
& \Leftrightarrow & & \left(c_{0}+c_{1}^{2}\right)^{2} & >4 c_{0} c_{1}^{2} \\
\Leftrightarrow & & c_{0}^{2}-2 c_{0} c_{1}^{2}+c_{1}^{4} & >0 \\
\Leftrightarrow & & & \left(c_{0}-c_{1}^{2}\right)^{2} & >0
\end{array}
$$

thus yielding the result.

Some of the above mentioned cases are (retrospectively) further subdivided to facilitate integration. An index of the final subdivision of cases is provided in Table 1.

In Figure 2, we graph the level sets of $H$ and $C$ and their intersection for each major case (i.e., by choosing some suitable values for $h_{0}, c_{0}, c_{1}$ and $c_{2}$ ). The stable equilibrium points (illustrated in blue) and unstable equilibrium points (illustrated in red), as presented in Theorem 4.2, are also plotted in each case.

We start our presentation of the integral curves of (3.5) by considering case 1a(i). (Formula (2.7) is utilised in the following theorem). 

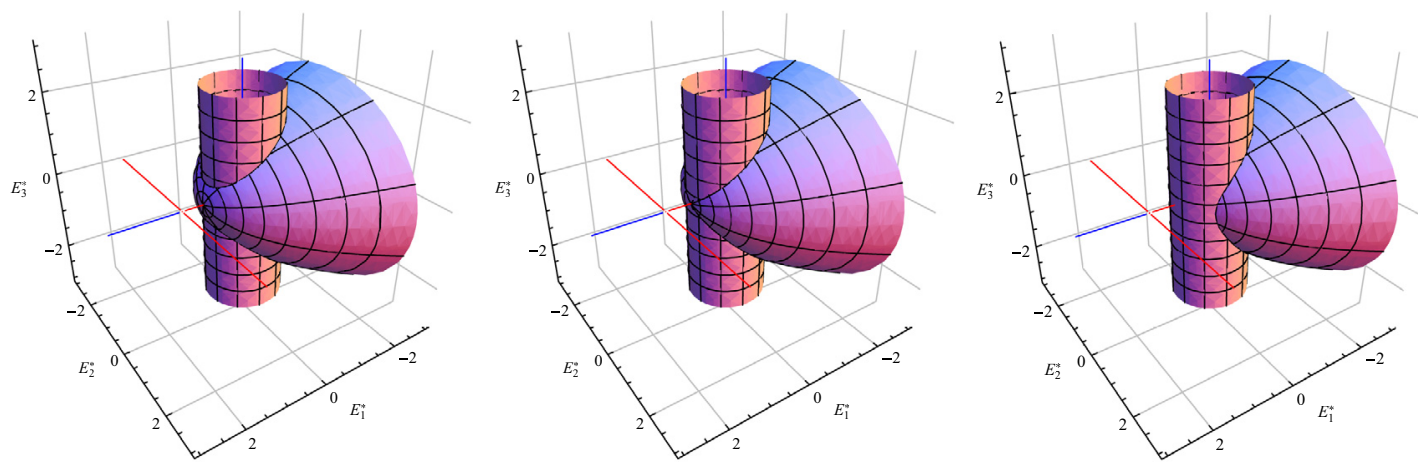

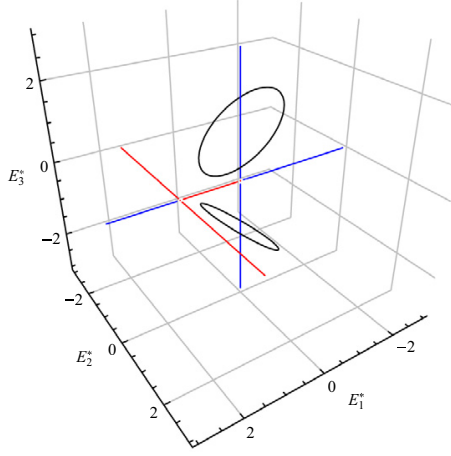

(A) Case 1a
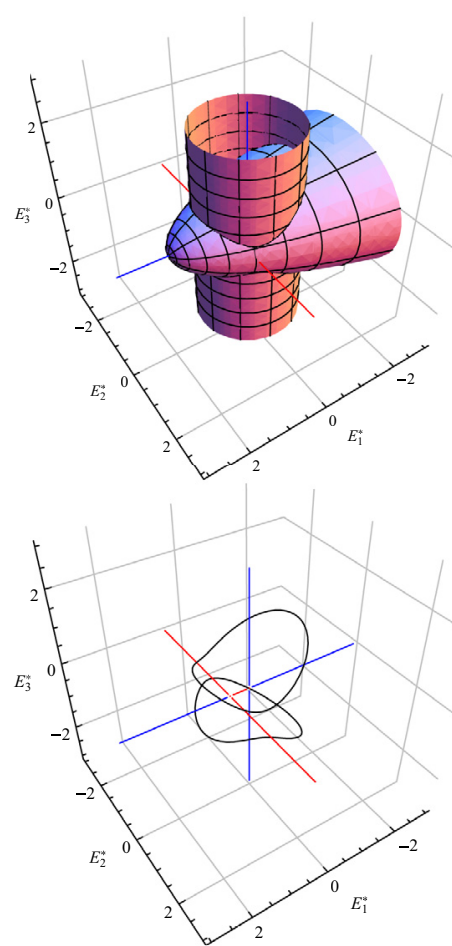

(D) Case $2 \mathrm{a}$

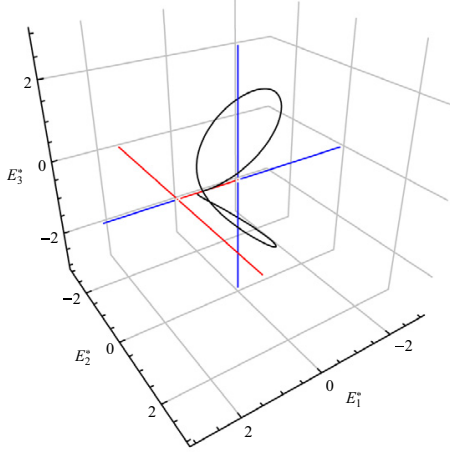

(B) Case 1b
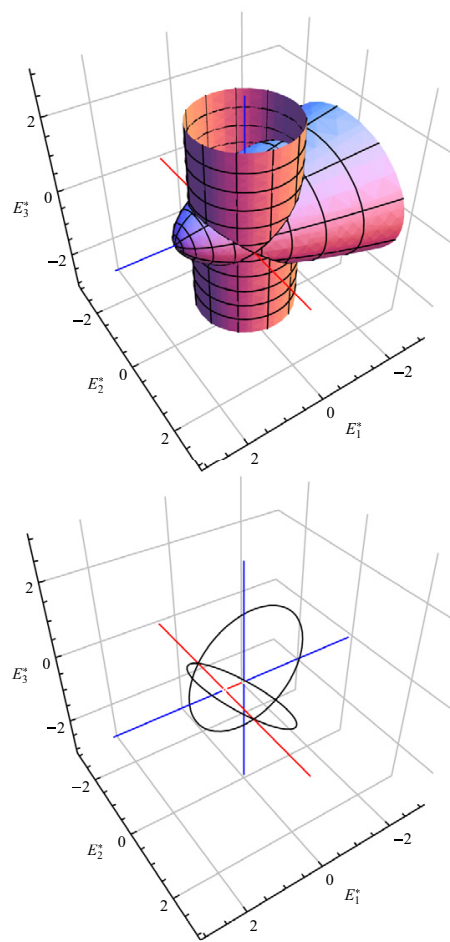

(E) Case $2 \mathrm{~b}$

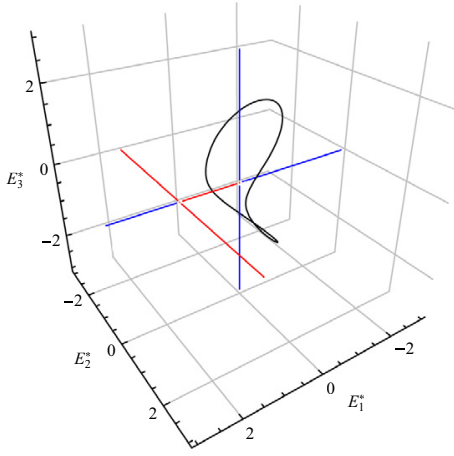

(c) Case 1c
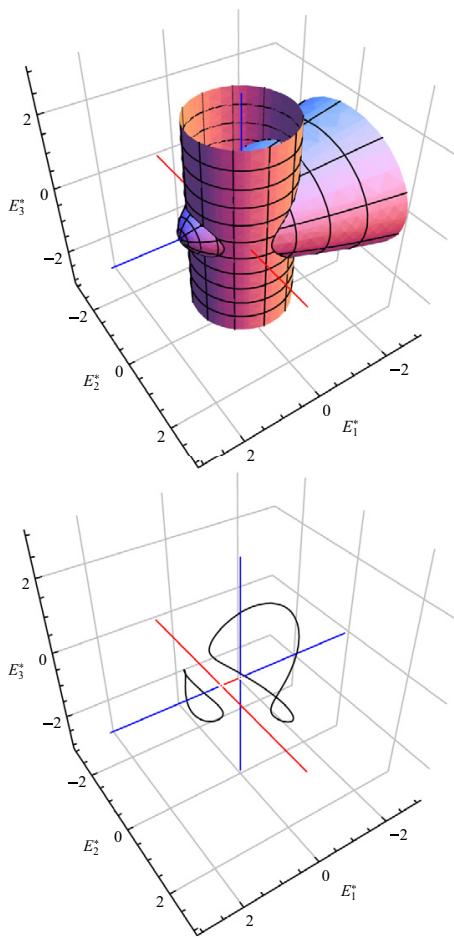

(F) Case 2c(i)

Figure 2. Typical cases of $\operatorname{LiCP}(2)$. 
TABLE 1. Index of typical cases for reduced extremals of LiCP(2).

\begin{tabular}{|c|c|c|c|}
\hline \multicolumn{3}{|c|}{ Conditions } & Index \\
\hline \multirow{6}{*}{$c_{0}-c_{1}^{2} \leq 0$} & \multirow{3}{*}{$h_{0}>\sqrt{c_{0}}$} & $c_{1}-h_{0}>\sqrt{h_{0}^{2}-c_{0}}$ & $1 \mathrm{a}(\mathrm{i})$ \\
\hline & & $c_{1}-h_{0}=\sqrt{h_{0}^{2}-c_{0}}$ & $1 \mathrm{a}(\mathrm{ii})$ \\
\hline & & $c_{1}-h_{0}<\sqrt{h_{0}^{2}-c_{0}}$ & $1 \mathrm{a}(\mathrm{iii})$ \\
\hline & \multirow{2}{*}{$h_{0}=\sqrt{c_{0}}$} & $h_{0}<c_{1}$ & $1 \mathrm{~b}(\mathrm{i})$ \\
\hline & & $h_{0}=c_{1}$ & $1 \mathrm{~b}(\mathrm{ii})$ \\
\hline & \multicolumn{2}{|c|}{$-\sqrt{c_{0}}<h_{0}<\sqrt{c_{0}}$} & $1 \mathrm{c}$ \\
\hline \multirow{5}{*}{$c_{0}-c_{1}^{2}>0$} & \multicolumn{2}{|c|}{$h_{0}>\frac{1}{2 c_{1}}\left(c_{0}+c_{1}^{2}\right)$} & $2 \mathrm{a}$ \\
\hline & \multicolumn{2}{|c|}{$h_{0}=\frac{1}{2 c_{1}}\left(c_{0}+c_{1}^{2}\right)$} & $2 \mathrm{~b}$ \\
\hline & \multirow{3}{*}{$h_{0}<\frac{c_{0}+c_{1}^{2}}{2 c_{1}}$} & $h_{0}>\sqrt{c_{0}}$ & $2 \mathrm{c}(\mathrm{i})$ \\
\hline & & $h_{0}=\sqrt{c_{0}}$ & $2 \mathrm{c}(\mathrm{ii})$ \\
\hline & & $-\sqrt{c_{0}}<h_{0}<\sqrt{c_{0}}$ & $2 \mathrm{c}(\mathrm{iii})$ \\
\hline
\end{tabular}

Theorem 5.7 (case 1a(i)). Suppose $p(\cdot):(-\varepsilon, \varepsilon) \rightarrow \mathfrak{s e}(2)^{*}$ is an integral curve of $\vec{H}$ such that $H(p(0))=h_{0}$, $C(p(0))=c_{0}>0$ and the conditions of case $1 a(i)$ are satisfied. Then there exists $t_{0} \in \mathbb{R}$ and $\sigma \in\{-1,1\}$ such that $p(t)=\bar{p}\left(t+t_{0}\right)$ for $t \in(-\varepsilon, \varepsilon)$, where

$$
\left\{\begin{array}{l}
\bar{p}_{1}(t)=\sqrt{c_{0}} \frac{\sqrt{h_{0}-\delta}-\sqrt{h_{0}+\delta} \operatorname{sn}(\Omega t, k)}{\sqrt{h_{0}+\delta}-\sqrt{h_{0}-\delta} \operatorname{sn}(\Omega t, k)} \\
\bar{p}_{2}(t)=-\sigma \sqrt{2 c_{0} \delta} \frac{\operatorname{cn}(\Omega t, k)}{\sqrt{h_{0}+\delta}-\sqrt{h_{0}-\delta} \operatorname{sn}(\Omega t, k)} \\
\bar{p}_{3}(t)=\frac{2 \sigma \delta \sqrt{c_{2}}}{k^{\prime}} \frac{\operatorname{dn}(\Omega t, k)}{\sqrt{h_{0}+\delta}-\sqrt{h_{0}-\delta} \operatorname{sn}(\Omega t, k)}
\end{array}\right.
$$

Here $\delta=\sqrt{h_{0}^{2}-c_{0}}, \Omega=\sqrt{\frac{\left(h_{0}+\delta\right)\left(c_{1}-h_{0}+\delta\right)}{c_{1} c_{2}}}, k=\sqrt{\frac{\left(h_{0}-\delta\right)\left(c_{1}-h_{0}-\delta\right)}{\left(h_{0}+\delta\right)\left(c_{1}-h_{0}+\delta\right)}}$ and $k^{\prime}=\sqrt{\frac{2 \delta c_{1}}{\left(h_{0}+\delta\right)\left(c_{1}-h_{0}+\delta\right)}}$.

Proof. Again, verification that $\bar{p}(\cdot)$ is a maximal integral curve satisfying $H(\bar{p}(0))=h_{0}$ and $C(p(0))=c_{0}$ whenever $c_{0}-c_{1}^{2} \leq 0, h_{0}>\sqrt{c_{0}}$ and $c_{1}-h_{0}>\sqrt{h_{0}^{2}-c_{0}}$ is straightforward. (Also, note that $\sqrt{h_{0}+\delta}>\sqrt{h_{0}-\delta}$ and so $\bar{p}(t)$ is defined for all $t \in \mathbb{R})$. We have $p_{1}(0)^{2}+p_{2}(0)^{2}=c_{0}, p_{1}(0)+\frac{1}{2}\left(\frac{1}{c_{1}} p_{2}(0)^{2}+\frac{1}{c_{2}} p_{3}(0)^{2}\right)=h_{0}$ and $h_{0}>\sqrt{c_{0}}$. Thus $-\sqrt{c_{0}} \leq p_{1}(0) \leq \sqrt{c_{0}}$. Also $p_{3}(0)^{2}=h_{0}-p_{1}(0)-\frac{1}{2 c_{1}}\left(c_{0}-p_{1}(0)^{2}\right)$. Now

$$
\min _{-\sqrt{c_{0}} \leq p_{1} \leq \sqrt{c_{0}}}\left(-p_{1}-\frac{1}{2 c_{1}}\left(c_{0}-p_{1}^{2}\right)\right)=-\sqrt{c_{0}} .
$$

Hence $p_{3}(0)^{2} \geq h_{0}-\sqrt{c_{0}}>0$. Hence $p_{3}(0) \neq 0$. Let $\sigma=\operatorname{sgn}\left(p_{3}(0)\right)$. Now $\bar{p}_{1}\left(-\frac{K}{\Omega}\right)=\sqrt{c_{0}}$ and $\bar{p}_{1}\left(\frac{K}{\Omega}\right)=-\sqrt{c_{0}}$. Thus there exists $t_{1} \in\left[-\frac{K}{\Omega}, \frac{K}{\Omega}\right]$ such that $\bar{p}_{1}\left(t_{1}\right)=p_{1}(0)$. Next

$$
p_{2}(0)^{2}=c_{0}-p_{1}(0)^{2}=c_{0}-\bar{p}_{1}\left(t_{1}\right)^{2}=\bar{p}_{2}\left(t_{1}\right)^{2} .
$$

Hence $p_{2}(0)= \pm \bar{p}_{2}\left(t_{1}\right)$. Now $\bar{p}_{1}\left(-t+\frac{2 K}{\Omega}\right)=\bar{p}_{1}(t)$ and $\bar{p}_{2}\left(-t+\frac{2 K}{\Omega}\right)=-\bar{p}_{2}(t)$. Thus there exists $t_{0} \in \mathbb{R}\left(t_{0}=t_{1}\right.$ or $\left.t_{0}=-t_{1}+\frac{2 K}{\Omega}\right)$ such that $p_{1}(0)=\bar{p}_{1}\left(t_{0}\right)$ and $p_{2}(0)=\bar{p}_{2}\left(t_{0}\right)$. We have

$$
p_{3}(0)^{2}=2 c_{2} h_{0}-2 c_{2} p_{1}(0)-\frac{c_{2}}{c_{1}} p_{2}(0)^{2}=2 c_{2} h_{0}-2 c_{2} \bar{p}_{1}\left(t_{0}\right)-\frac{c_{2}}{c_{1}} \bar{p}_{2}\left(t_{0}\right)^{2}=\bar{p}_{3}\left(t_{0}\right)^{2} .
$$

Thus, as $\operatorname{sgn}\left(p_{3}(0)\right)=\sigma=\operatorname{sgn}\left(\bar{p}_{3}\left(t_{0}\right)\right)$, we get $p_{3}(0)=\bar{p}_{3}\left(t_{0}\right)$. Hence $p(0)=\bar{p}\left(t_{0}\right)$. Consequently the integral curves $t \mapsto p(t)$ and $t \mapsto \bar{p}\left(t+t_{0}\right)$ solve the same Cauchy problem, and therefore are identical. 
Next, by limiting $\delta \rightarrow c_{1}-h_{0}$ (from the right) in the above result, we get a prospective integral curve for case $1 \mathrm{a}$ (ii). The proof is omitted as it is essentially the same as for Theorem 5.7 ( $K$ is replaced by $\left.\frac{\pi}{2}\right)$.

Proposition 5.8 (case 1a(ii)). Suppose $p(\cdot):(-\varepsilon, \varepsilon) \rightarrow \mathfrak{s e}(2)^{*}$ is an integral curve of $\vec{H}$ such that $H(p(0))=$ $h_{0}, C(p(0))=c_{0}>0$ and the conditions of case $1 a\left(\right.$ ii) are satisfied. Then there exists $t_{0} \in \mathbb{R}$ and $\sigma \in\{-1,1\}$ such that $p(t)=\bar{p}\left(t+t_{0}\right)$ for $t \in(-\varepsilon, \varepsilon)$, where

$$
\left\{\begin{array}{l}
\bar{p}_{1}(t)=\sqrt{c_{0}} \frac{\sqrt{h_{0}-\delta}-\sqrt{h_{0}+\delta} \sin (\Omega t)}{\sqrt{h_{0}+\delta}-\sqrt{h_{0}-\delta} \sin (\Omega t)} \\
\bar{p}_{2}(t)=-\sigma \sqrt{2 c_{0} \delta} \frac{\cos (\Omega t)}{\sqrt{h_{0}+\delta}-\sqrt{h_{0}-\delta} \sin (\Omega t)} \\
\bar{p}_{3}(t)=\frac{2 \sigma \delta \sqrt{c_{2}}}{\sqrt{h_{0}+\delta}-\sqrt{h_{0}-\delta} \sin (\Omega t)} .
\end{array}\right.
$$

Here $\delta=\sqrt{h_{0}^{2}-c_{0}}$ and $\Omega=\sqrt{\frac{2\left(c_{1}-h_{0}\right)}{c_{2}}}$.

It turns out that integral curves satisfying the conditions of case 1a(iii) or 2a take the same explicit expression. We shall provide a detailed proof for the result.

Lemma 5.9. For cases $1 a($ iii) and $2 a$ we have

$$
h_{0}-c_{1}+\sqrt{h_{0}^{2}-c_{0}}>0 \quad \text { and } \quad c_{1}-h_{0}+\sqrt{h_{0}^{2}-c_{0}}>0 .
$$

Theorem 5.10 (cases $1 \mathrm{a}(\mathrm{iii})$ and $2 \mathrm{a})$. Suppose $p(\cdot):(-\varepsilon, \varepsilon) \rightarrow \mathfrak{s e}(2)^{*}$ is an integral curve of $\vec{H}$ such that $H(p(0))=h_{0}, C(p(0))=c_{0}>0$ and the conditions of case $1 a(\mathrm{iii})$ or $2 a$ are satisfied. Then there exists $t_{0} \in \mathbb{R}$ and $\sigma \in\{-1,1\}$ such that $p(t)=\bar{p}\left(t+t_{0}\right)$ for $t \in(-\varepsilon, \varepsilon)$, where

$$
\left\{\begin{array}{l}
\bar{p}_{1}(t)=\sqrt{c_{0}} \frac{\sqrt{h_{0}-\delta}-\sqrt{h_{0}+\delta} \operatorname{cn}(\Omega t, k)}{\sqrt{h_{0}+\delta}-\sqrt{h_{0}-\delta} \operatorname{cn}(\Omega t, k)} \\
\bar{p}_{2}(t)=\sigma \sqrt{2 c_{0} \delta} \frac{\operatorname{sn}(\Omega t, k)}{\sqrt{h_{0}+\delta}-\sqrt{h_{0}-\delta} \operatorname{cn}(\Omega t, k)} \\
\bar{p}_{3}(t)=2 \sigma \delta \sqrt{c_{2}} \frac{\operatorname{dn}(\Omega t, k)}{\sqrt{h_{0}+\delta}-\sqrt{h_{0}-\delta} \operatorname{cn}(\Omega t, k)}
\end{array}\right.
$$

Here $\delta=\sqrt{h_{0}^{2}-c_{0}}, \Omega=\sqrt{\frac{2 \delta}{c_{2}}}$ and $k=\sqrt{\frac{\left(h_{0}-\delta\right)\left(h_{0}-c_{1}+\delta\right)}{2 \delta c_{1}}}$.

Proof. We start by explaining how the expression for $\bar{p}(\cdot)$ was found. A number of convenient assumptions are made implicitly and translations in the independent variable are discarded. We shall verify that $\bar{p}(\cdot)$ is a maximal integral curve (defined for any $h_{0}, c_{0}, c_{1}$ and $c_{2}$ satisfying the conditions of case 1a(iii) or 2a) only at the end of the construction.

Suppose $\bar{p}(\cdot)$ is an integral curve of $\vec{H}$ satisfying the conditions of $1 \mathrm{a}$ (iii) or $2 \mathrm{a}$, where $h_{0}=H(\bar{p}(0))$ and $c_{0}=C(\bar{p}(0))$. We transform (5.2) into standard form (see, e.g., [4] or [15]). First, we can rewrite (5.2) as

$$
\frac{\mathrm{d} \bar{p}_{1}}{\mathrm{~d} t}=\sqrt{\left(A_{1}\left(\bar{p}_{1}-r_{1}\right)^{2}+B_{1}\left(\bar{p}_{1}-r_{2}\right)^{2}\right)\left(A_{2}\left(\bar{p}_{1}-r_{1}\right)^{2}+B_{2}\left(\bar{p}_{1}-r_{2}\right)^{2}\right)}
$$

where

$$
\begin{aligned}
A_{1} & =\frac{h_{0}+\delta}{2 c_{1} c_{2} \delta}>0 & A_{2} & =-\frac{h_{0}-c_{1}+\delta}{2 \delta}<0 \\
B_{1} & =-\frac{h_{0}-\delta}{2 c_{1} c_{2} \delta}<0 & B_{2} & =-\frac{c_{1}-h_{0}+\delta}{2 \delta}<0 \\
r_{1} & =h_{0}-\delta & r_{2} & =h_{0}+\delta .
\end{aligned}
$$


Then we have

$$
\int \mathrm{d} t=\int \frac{\mathrm{d} \bar{p}_{1}}{\sqrt{\left(A_{1}\left(\bar{p}_{1}-r_{1}\right)^{2}+B_{1}\left(\bar{p}_{1}-r_{2}\right)^{2}\right)\left(A_{2}\left(\bar{p}_{1}-r_{1}\right)^{2}+B_{2}\left(\bar{p}_{1}-r_{2}\right)^{2}\right)}} .
$$

We make a change of variable $s=\frac{\bar{p}_{1}-r_{1}}{\bar{p}_{1}-r_{2}}$, which then yields

$$
t=\frac{1}{\left(r_{1}-r_{2}\right) \sqrt{-A_{1} A_{2}}} \int_{0}^{\frac{\bar{p}_{1}(t)-r_{1}}{\bar{p}_{1}(t)-r_{2}}} \frac{d s}{\left(\frac{B_{2}}{A_{2}}+s^{2}\right)\left(-\frac{B_{1}}{A_{1}}-s^{2}\right)} .
$$

Applying formula (2.8), we get

$$
\left(r_{1}-r_{2}\right) \sqrt{-A_{1} A_{2}} t=\frac{1}{\sqrt{\frac{B_{2}}{A_{2}}-\frac{B_{1}}{A_{1}}}} \operatorname{sd}^{-1}\left(\frac{\sqrt{\frac{B_{2}}{A_{2}}-\frac{B_{1}}{A_{1}}}}{\sqrt{-\frac{B_{1}}{A_{1}}} \sqrt{\frac{B_{2}}{A_{2}}}} \frac{\bar{p}_{1}(t)-r_{1}}{\bar{p}_{1}(t)-r_{2}}, \frac{\sqrt{-\frac{B_{1}}{A_{1}}}}{\sqrt{\frac{B_{2}}{A_{2}}-\frac{B_{1}}{A_{1}}}}\right) .
$$

Solving for $\bar{p}_{1}(t)$ we find

$$
\bar{p}_{1}(t)=\frac{r_{2} \sqrt{\frac{B_{1} B_{2}}{B_{1} A_{2}-B_{2} A_{1}}} \operatorname{sd}\left(\left(r_{1}-r_{2}\right) \sqrt{B_{1} A_{2}-B_{2} A_{1}} t, \sqrt{\frac{B_{1} A_{2}}{B_{1} A_{2}-B_{2} A_{1}}}\right)-r_{1}}{\sqrt{\frac{B_{1} B_{2}}{B_{1} A_{2}-B_{2} A_{1}}} \operatorname{sd}\left(\left(r_{1}-r_{2}\right) \sqrt{B_{1} A_{2}-B_{2} A_{1}} t, \sqrt{\frac{B_{1} A_{2}}{B_{1} A_{2}-B_{2} A_{1}}}\right)-1} .
$$

Substituting the values for $A_{1}, A_{2}, B_{1}, B_{2}, r_{1}, r_{2}$ and simplifying then yields

$$
\bar{p}_{1}(t)=\sqrt{h_{0}^{2}-\delta^{2}} \frac{\sqrt{h_{0}-\delta}-\sqrt{h_{0}+\delta} k^{\prime} \operatorname{sd}(\Omega t, k)}{\sqrt{h_{0}+\delta}-\sqrt{h_{0}-\delta} k^{\prime} \operatorname{sd}(\Omega t, k)} .
$$

Now $\operatorname{cn}(x+3 K, k)=k^{\prime} \mathrm{sd}(x, k)$. Thus, by making a suitable translation in $t$, we get the following (prospective) expression for $\bar{p}_{1}$

$$
\bar{p}_{1}(t)=\sqrt{h_{0}^{2}-\delta^{2}} \frac{\sqrt{h_{0}-\delta}-\sqrt{h_{0}+\delta} \operatorname{cn}(\Omega t, k)}{\sqrt{h_{0}+\delta}-\sqrt{h_{0}-\delta} \operatorname{cn}(\Omega t, k)} .
$$

Next, as $C(\bar{p}(t))=c_{0}$, we get

$$
\bar{p}_{2}(t)^{2}=c_{0}-\bar{p}_{1}(t)^{2}=\frac{2 \delta\left(h_{0}^{2}-\delta^{2}\right) \operatorname{sn}(\Omega t, k)^{2}}{\left(\sqrt{h_{0}+\delta}-\sqrt{h_{0}-\delta} \operatorname{cn}(\Omega t, k)\right)^{2}} .
$$

Hence

$$
\bar{p}_{2}(t)=\sigma_{2} \frac{\sqrt{2 c_{0} \delta} \operatorname{sn}(\Omega t, k)}{\sqrt{h_{0}+\delta}-\sqrt{h_{0}-\delta} \operatorname{cn}(\Omega t, k)}
$$

for some $\sigma_{2} \in\{-1,1\}$. By a similar computation, using the constant of motion $H$, we obtain

$$
\bar{p}_{3}(t)=\sigma_{3} \frac{2 \delta \sqrt{c_{2}} \operatorname{dn}(\Omega t, k)}{\sqrt{h_{0}+\delta}-\sqrt{h_{0}-\delta} \operatorname{cn}(\Omega t, k)}
$$

for some $\sigma_{3} \in\{-1,1\}$. We now show that $\bar{p}(\cdot)$ is an integral curve for certain values of $\sigma_{2}$ and $\sigma_{3}$. From (3.5), we require that $\frac{\mathrm{d}}{\mathrm{d} t} \bar{p}_{1}(t)=\frac{1}{c_{2}} \bar{p}_{2}(t) \bar{p}_{3}(t)$. Now

$$
\frac{\mathrm{d}}{\mathrm{d} t} \bar{p}_{1}(t)-\frac{1}{c_{2}} \bar{p}_{2}(t) \bar{p}_{3}(t)=\frac{2 \delta \sqrt{2 \delta} \mathrm{dn}(\Omega t, k) \operatorname{sn}(\Omega t, k) \sqrt{\frac{h_{0}^{2}-\delta^{2}}{c_{2}}}\left(1-\sigma_{2} \sigma_{3}\right)}{\left(\sqrt{h_{0}+\delta}-\sqrt{h_{0}-\delta} \operatorname{cn}(\Omega t, k)\right)^{2}} .
$$


Therefore, if $\sigma_{2}=\sigma_{3}=\sigma \in\{-1,1\}$, then $\frac{\mathrm{d}}{\mathrm{d} t} \bar{p}_{1}(t)=\frac{1}{c_{2}} \bar{p}_{2}(t) \bar{p}_{3}(t)$. For this choice of $\sigma_{2}$ and $\sigma_{3}$, further calculation shows that $\frac{\mathrm{d}}{\mathrm{d} t} \bar{p}_{2}(t)=-\frac{1}{c_{2}} \bar{p}_{1}(t) \bar{p}_{3}(t)$ and $\frac{\mathrm{d}}{\mathrm{d} t} \bar{p}_{3}(t)=\left(\frac{1}{c_{1}} \bar{p}_{1}(t)-1\right) \bar{p}_{2}(t)$. This motivates $\bar{p}(\cdot)$ as a prospective integral curve.

Elementary calculations show that $\bar{p}(\cdot)$ is defined over $\mathbb{R}$ when either set of conditions (case 1a(iii) or $2 \mathrm{a}$ ) is satisfied. In particular, note that the denominator in each expression is strictly positive, the constants $\delta, k$ and $\Omega$ are real, and $0<k<1$. Thus $\bar{p}(\cdot)$ is a maximal integral curve of $\vec{H}$ for any $h_{0}, c_{0}, c_{1}$ and $c_{2}$ satisfying the conditions of case $1 \mathrm{a}(\mathrm{iii})$ or $2 \mathrm{a}$.

Finally, we claim that any integral curve $p(\cdot)$ (as described in the statement of the theorem) must be of the form $p(t)=\bar{p}\left(t+t_{0}\right)$ for some $\sigma \in\{-1,1\}$ and $t_{0} \in \mathbb{R}$. By Theorem 4.6, $\bar{p}(\cdot)$ covers each connected component of $H^{-1}\left(h_{0}\right) \cap C^{-1}\left(c_{0}\right)$ (for each $\sigma \in\{-1,1\}$, respectively) as illustrated in Figures 2a and $2 \mathrm{~d}$. Thus, as $p(0) \in H^{-1}\left(h_{0}\right) \cap C^{-1}\left(c_{0}\right)$ by assumption, there must exists $t_{0} \in \mathbb{R}$ and $\sigma \in\{-1,1\}$ satisfying the conditions of the theorem. We now give a proof for this claim.

We have $p_{1}(0)^{2}+p_{2}(0)^{2}=c_{0}$ and $p_{1}(0)+\frac{1}{2}\left(\frac{1}{c_{1}} p_{2}(0)^{2}+\frac{1}{c_{2}} p_{3}(0)^{2}\right)=h_{0}$. Thus $-\sqrt{c_{0}} \leq p_{1}(0) \leq \sqrt{c_{0}}$. Also $\frac{1}{2 c_{2}} p_{3}(0)^{2}=h_{0}-p_{1}(0)-\frac{1}{2 c_{1}}\left(c_{0}-p_{1}(0)^{2}\right)$. Suppose the conditions of case 1a(iii) hold. Then

$$
\min _{-\sqrt{c_{0}} \leq p_{1} \leq \sqrt{c_{0}}}\left(-p_{1}-\frac{1}{2 c_{1}}\left(c_{0}-p_{1}^{2}\right)\right)=-\sqrt{c_{0}} .
$$

Hence $\frac{1}{2 c_{2}} p_{3}(0)^{2} \geq h_{0}-\sqrt{c_{0}}>0$ and so $p_{3}(0) \neq 0$. On the other hand, suppose the conditions of case 2 a hold. Then

$$
\min _{-\sqrt{c_{0}} \leq p_{1} \leq \sqrt{c_{0}}}\left(-p_{1}-\frac{1}{2 c_{1}}\left(c_{0}-p_{1}^{2}\right)\right)=-\frac{1}{2 c_{1}}\left(c_{0}+c_{1}^{2}\right) .
$$

Hence $\frac{1}{2 c_{2}} p_{3}(0)^{2} \geq h_{0}-\frac{1}{2 c_{1}}\left(c_{0}+c_{1}^{2}\right)>0$ and so $p_{3}(0) \neq 0$.

Let $\sigma=\operatorname{sgn}\left(p_{3}(0)\right)$. Now $\bar{p}_{1}(0)=-\sqrt{c_{0}}$ and $\bar{p}_{1}\left(\frac{2 K}{\Omega}\right)=\sqrt{c_{0}}$. Thus there exists $t_{1} \in\left[0, \frac{2 K}{\Omega}\right]$ such that $\bar{p}_{1}\left(t_{1}\right)=p_{1}(0)$. Next we have

$$
p_{2}(0)^{2}=c_{0}-p_{1}(0)^{2}=c_{0}-\bar{p}_{1}\left(t_{1}\right)^{2}=\bar{p}_{2}\left(t_{1}\right)^{2} .
$$

Therefore $p_{2}(0)= \pm \bar{p}_{2}\left(t_{1}\right)$. Now $\bar{p}_{1}(-t)=\bar{p}_{1}(t)$ and $\bar{p}_{2}(-t)=-\bar{p}_{2}(t)$. Hence there exists $t_{0} \in \mathbb{R}\left(t_{0}=t_{1}\right.$ or $\left.t_{0}=-t_{1}\right)$ such that $p_{1}(0)=\bar{p}_{1}\left(t_{0}\right)$ and $p_{2}(0)=\bar{p}_{2}\left(t_{0}\right)$. Next

$$
p_{3}(0)^{2}=2 c_{2} h_{0}-2 c_{2} p_{1}(0)-\frac{c_{2}}{c_{1}} p_{2}(0)^{2}=2 c_{2} h_{0}-2 c_{2} \bar{p}_{1}\left(t_{0}\right)-\frac{c_{2}}{c_{1}} \bar{p}_{2}\left(t_{0}\right)^{2}=\bar{p}_{3}\left(t_{0}\right)^{2} .
$$

Thus, as $\operatorname{sgn}\left(p_{3}(0)\right)=\sigma=\operatorname{sgn}\left(\bar{p}_{3}\left(t_{0}\right)\right)$, we get $p_{3}(0)=\bar{p}_{3}\left(t_{0}\right)$. Hence $p(0)=\bar{p}\left(t_{0}\right)$. Consequently the integral curves $t \mapsto p(t)$ and $t \mapsto \bar{p}\left(t+t_{0}\right)$ solve the same Cauchy problem, and therefore are identical.

Limiting processes were unsuccessful in producing a prospective integral curve for case $1 \mathrm{~b}$. Therefore, we resorted to integrating case $1 \mathrm{~b}(\mathrm{i})$ independently by use of formula $(2.9)$.

Proposition 5.11 (case $1 \mathrm{~b}(\mathrm{i}))$. Suppose $p(\cdot):(-\varepsilon, \varepsilon) \rightarrow \mathfrak{s e}(2)^{*}$ is an integral curve of $\vec{H}$ such that $H(p(0))=$ $h_{0}, C(p(0))=c_{0}>0$ and the conditions of case $1 b(i)$ are satisfied. Then there exists $t_{0} \in \mathbb{R}$ and $\sigma \in\{-1,1\}$ such that $p(t)=\bar{p}\left(t+t_{0}\right)$ for $t \in(-\varepsilon, \varepsilon)$, where

$$
\left\{\begin{array}{l}
\bar{p}_{1}(t)=\frac{h_{0}}{2} \frac{c_{1}(\cosh (2 \Omega t)-3)+2 h_{0}}{c_{1} \cosh ^{2}(\Omega t)-h_{0}} \\
\bar{p}_{2}(t)=2 \sigma h_{0} \sqrt{c_{1}\left(c_{1}-h_{0}\right)} \frac{\sinh (\Omega t)}{c_{1} \cosh ^{2}(\Omega t)-h_{0}} \\
\bar{p}_{3}(t)=2 \sigma\left(c_{1}-h_{0}\right) \sqrt{c_{2} h_{0}} \frac{\cosh (\Omega t)}{c_{1} \cosh ^{2}(\Omega t)-h_{0}} .
\end{array}\right.
$$

Here $\Omega=\sqrt{\frac{h_{0}\left(c_{1}-h_{0}\right)}{c_{1} c_{2}}}$. 
Proof. We have $p_{1}(0)^{2}+p_{2}(0)^{2}=c_{0}, p_{1}(0)+\frac{1}{2}\left(\frac{1}{c_{1}} p_{2}(0)^{2}+\frac{1}{c_{2}} p_{3}(0)^{2}\right)=h_{0}$ and $h_{0}=\sqrt{c_{0}}$. If $p_{3}(0)=0$, then a simple calculation shows that $p_{2}(0)=0$ and so $p(\cdot)$ is constant. Hence $p_{3}(0) \neq 0$ (if $p(\cdot)$ is not constant). Let $\sigma=\operatorname{sgn}\left(p_{3}(0)\right)$.

Now $-\sqrt{c_{0}} \leq p_{1}(0)<\sqrt{c_{0}}, \bar{p}_{1}(0)=-\sqrt{c_{0}}$ and $\lim _{t \rightarrow \infty} \bar{p}_{1}(t)=\sqrt{c_{0}}$. Thus there exists $t_{1} \in \mathbb{R}$ such that $\bar{p}_{1}\left(t_{1}\right)=p_{1}(0)$. Next

$$
p_{2}(0)^{2}=c_{0}-p_{1}(0)^{2}=c_{0}-\bar{p}_{1}\left(t_{1}\right)^{2}=\bar{p}_{2}\left(t_{1}\right)^{2} .
$$

Hence $p_{2}(0)= \pm \bar{p}_{2}\left(t_{1}\right)$. Now $\bar{p}_{1}(-t)=\bar{p}_{1}(t)$ and $\bar{p}_{2}(-t)=-\bar{p}_{2}(t)$. Thus there exists $t_{0} \in \mathbb{R}\left(t_{0}=t_{1}\right.$ or $\left.t_{0}=-t_{1}\right)$ such that $p_{1}(0)=\bar{p}_{1}\left(t_{0}\right)$ and $p_{2}(0)=\bar{p}_{2}\left(t_{0}\right)$. We have

$$
p_{3}(0)^{2}=2 c_{2} h_{0}-2 c_{2} p_{1}(0)-\frac{c_{2}}{c_{1}} p_{2}(0)^{2}=2 c_{2} h_{0}-2 c_{2} \bar{p}_{1}\left(t_{0}\right)-\frac{c_{2}}{c_{1}} \bar{p}_{2}\left(t_{0}\right)^{2}=\bar{p}_{3}\left(t_{0}\right)^{2} .
$$

Thus, as $\operatorname{sgn}\left(p_{3}(0)\right)=\sigma=\operatorname{sgn}\left(\bar{p}_{3}\left(t_{0}\right)\right)$, we get $p_{3}(0)=\bar{p}_{3}\left(t_{0}\right)$. Hence $p(0)=\bar{p}\left(t_{0}\right)$. Consequently the integral curves $t \mapsto p(t)$ and $t \mapsto \bar{p}\left(t+t_{0}\right)$ solve the same Cauchy problem, and therefore are identical.

A simple calculation (either limiting $h_{0} \rightarrow c_{1}$ from the left in the above case, or integrating (5.2) directly) then yields a prospective integral curve for case $1 \mathrm{~b}(\mathrm{ii})$. The proof is omitted as it is essentially the same as for Proposition 5.11.

Proposition 5.12 (case $1 \mathrm{~b}(\mathrm{ii}))$. Suppose $p(\cdot):(-\varepsilon, \varepsilon) \rightarrow \mathfrak{s e}(2)^{*}$ is an integral curve of $\vec{H}$ such that $H(p(0))=$ $h_{0}, C(p(0))=c_{0}>0$ and the conditions of case $1 b(\mathrm{ii})$ are satisfied. Then there exists $t_{0} \in \mathbb{R}$ and $\sigma \in\{-1,1\}$ such that $p(t)=\bar{p}\left(t+t_{0}\right)$ for $t \in(-\varepsilon, \varepsilon)$, where

$$
\left\{\begin{array}{l}
\bar{p}_{1}(t)=c_{1} \frac{c_{1} t^{2}-c_{2}}{c_{1} t^{2}+c_{2}} \\
\bar{p}_{2}(t)=2 \sigma c_{1} \sqrt{c_{1} c_{2}} \frac{t}{c_{1} t^{2}+c_{2}} \\
\bar{p}_{3}(t)=2 \sigma c_{2} \sqrt{c_{1} c_{2}} \frac{1}{c_{1} t^{2}+c_{2}} .
\end{array}\right.
$$

For case 1c, the roots of the two quadratics in (5.2) need to be deinterlaced before this differential equation can be transformed into standard form. Consequently, the expression for this integral curve is quite involved. It turns out that integral curves satisfying the conditions of case 2c(iii), take the same explicit expression as those of case 1c. (The following result utilises formula (2.7)).

Theorem 5.13 (cases $1 \mathrm{c}$ and $2 \mathrm{c}(\mathrm{iii})$ ). Suppose $p(\cdot):(-\varepsilon, \varepsilon) \rightarrow \mathfrak{s e}(2)^{*}$ is an integral curve of $\vec{H}$ such that $H(p(0))=h_{0}, C(p(0))=c_{0}>0$ and the conditions of case $1 c$ or $2 c$ (iii) are satisfied. Then there exists $t_{0} \in \mathbb{R}$ such that $p(t)=\bar{p}\left(t+t_{0}\right)$ for $t \in(-\varepsilon, \varepsilon)$, where

$$
\left\{\begin{array}{l}
\bar{p}_{1}(t)=\eta_{1} \frac{\frac{2 \sqrt{c_{0}} c_{1}-\rho_{2}}{2 \sqrt{c_{0}} c_{1}+\rho_{2}} \sqrt{\rho_{1}+\rho_{2}}-\sqrt{\rho_{1}-\rho_{2}} \operatorname{cd}(\Omega t, k)}{\sqrt{\rho_{1}+\rho_{2}}-\sqrt{\rho_{1}-\rho_{2}} \operatorname{cd}(\Omega t, k)} \\
\bar{p}_{2}(t)=-\frac{\eta_{2} k^{\prime}}{\sqrt{k}} \frac{\operatorname{sd}\left(\frac{1}{2} \Omega t, k\right) \sqrt{1+\operatorname{nd}(\Omega t, k)} \sqrt{1+k \operatorname{cd}(\Omega t, k)}}{\sqrt{\rho_{1}+\rho_{2}}-\sqrt{\rho_{1}-\rho_{2}} \operatorname{cd}(\Omega t, k)} \\
\bar{p}_{3}(t)=-\frac{\eta_{3}}{\sqrt{k}} \frac{\operatorname{cn}\left(\frac{1}{2} \Omega t, k\right) \sqrt{1+\operatorname{nd}(\Omega t, k)} \sqrt{1-k \operatorname{cd}(\Omega t, k)}}{\sqrt{\rho_{1}+\rho_{2}}-\sqrt{\rho_{1}-\rho_{2}} \operatorname{cd}(\Omega t, k)}
\end{array}\right.
$$


Here

$$
\begin{aligned}
\delta & =\sqrt{c_{1}^{2}-2 c_{1} h_{0}+c_{0}} & k & =\sqrt{\frac{\rho_{1}-\frac{1}{2}\left(\sqrt{c_{0}}+c_{1}-\delta\right)^{2}-\rho_{2}}{\rho_{1}-\frac{1}{2}\left(\sqrt{c_{0}}+c_{1}-\delta\right)^{2}+\rho_{2}}} \\
\rho_{1} & =\left(c_{1}+2 \delta\right) \sqrt{c_{0}}+\delta\left(\delta-c_{1}\right)+c_{0} & k^{\prime} & =\sqrt{\frac{2 \rho_{2}}{\rho_{1}-\frac{1}{2}\left(\sqrt{c_{0}}+c_{1}-\delta\right)^{2}+\rho_{2}}} \\
\rho_{2} & =2 \sqrt[4]{c_{0}} \sqrt{\delta\left(\left(\sqrt{c_{0}}+\delta\right)^{2}-c_{1}^{2}\right)} & \Omega & =\sqrt{\frac{\rho_{1}-\frac{1}{2}\left(\sqrt{c_{0}}+c_{1}-\delta\right)^{2}+\rho_{2}}{2 c_{1} c_{2}}}
\end{aligned}
$$

and

$$
\begin{aligned}
& \eta_{1}=\frac{\left(2 c_{1} \sqrt{c_{0}}+\rho_{2}\right)}{2\left(\delta+\sqrt{c_{0}}\right)} \\
& \eta_{2}=\sqrt{\sqrt{c_{0}}\left(\sqrt{c_{0}}+c_{1}-\delta\right)\left(\rho_{2}+\left(\sqrt{c_{0}}+c_{1}-\delta\right) c_{1}-\rho_{1}\right)} \\
& \eta_{3}=\sqrt{\frac{c_{2}\left(\rho_{1}-\rho_{2}\right) \rho_{2}\left(4 \delta c_{1}+\left(\frac{\delta}{\sqrt{c_{0}}}-1\right) \rho_{2}\right)}{4\left(\delta+\sqrt{c_{0}}\right)^{2} c_{1}}} .
\end{aligned}
$$

Proof. Verification that $\bar{p}(\cdot)$ is an integral curve is quite involved. However, it is sufficient to show that $\dot{\bar{p}}_{1}(t)=$ $\frac{1}{c_{2}} \bar{p}_{2}(t) \bar{p}_{3}(t), \bar{p}_{1}(t)^{2}+\bar{p}_{2}(t)^{2}=c_{0}$ and $\bar{p}_{1}(t)+\frac{1}{2}\left(\frac{1}{c_{1}} \bar{p}_{2}(t)^{2}+\frac{1}{c_{2}} \bar{p}_{3}(t)^{2}\right)=h_{0}$.

We have $p_{1}(0)^{2}+p_{2}(0)^{2}=c_{0}$ and $p_{1}(0)+\frac{1}{2}\left(\frac{1}{c_{1}} p_{2}(0)^{2}+\frac{1}{c_{2}} p_{3}(0)^{2}\right)=h_{0}$. Hence $-\sqrt{c_{0}} \leq p_{1}(0) \leq \sqrt{c_{0}}$. Also, $p_{1}(0)+\frac{1}{2 c_{1}}\left(c_{0}-p_{1}(0)^{2}\right)-h_{0} \leq 0$. Thus $\frac{1}{2 c_{1}}\left(p_{1}(0)-\left(c_{1}-\delta\right)\right)\left(p_{1}(0)-\left(c_{1}+\delta\right)\right) \geq 0$, and so $p_{1}(0) \leq c_{1}-\delta$ or $p_{1}(0) \geq c_{1}+\delta$. It turns out that $-\sqrt{c_{0}} \leq c_{1}-\delta \leq \sqrt{c_{0}} \leq c_{1}+\delta$. Therefore $-\sqrt{c_{0}} \leq p_{1}(0) \leq c_{1}-\delta$.

We have that

$$
\begin{aligned}
& \bar{p}_{1}(-t)=\bar{p}_{1}(t) \\
& \bar{p}_{1}\left(t+\frac{4 K}{\Omega}\right)=\bar{p}_{1}(t) \\
& \bar{p}_{2}(-t)=-\bar{p}_{2}(t) \\
& \bar{p}_{2}\left(t+\frac{4 K}{\Omega}\right)=-\bar{p}_{2}(t) \\
& \bar{p}_{1}\left(-t+\frac{4 K}{\Omega}\right)=\bar{p}_{1}(t) \\
& \bar{p}_{3}(-t)=\bar{p}_{3}(t) \\
& \bar{p}_{3}\left(t+\frac{4 K}{\Omega}\right)=-\bar{p}_{3}(t) \\
& \bar{p}_{2}\left(-t+\frac{4 K}{\Omega}\right)=\bar{p}_{2}(t) \\
& \bar{p}_{3}\left(-t+\frac{4 K}{\Omega}\right)=-\bar{p}_{3}(t) .
\end{aligned}
$$

Now $\bar{p}_{1}(0)=-\sqrt{c_{0}}$ and $\bar{p}_{1}\left(\frac{2 K}{\Omega}\right)=c_{1}-\delta$. Hence there exists $t_{1} \in\left[0, \frac{2 K}{\Omega}\right]$ such that $\bar{p}_{1}\left(t_{1}\right)=p_{1}(0)$. Then (by using the constants of motion $C$ and $H)$ we get $p_{2}(0)= \pm \bar{p}_{2}\left(t_{1}\right)$ and $p_{3}(0)= \pm \bar{p}_{3}\left(t_{1}\right)$. Therefore there exists $t_{0} \in \mathbb{R}\left(t_{0}=t_{1}, t_{0}=-t_{1}, t_{0}=t_{1}+\frac{2 K}{\Omega}\right.$, or $\left.t_{0}=-t_{1}+\frac{2 K}{\Omega}\right)$ such that $p(0)=\bar{p}\left(t_{0}\right)$. Consequently the integral curves $t \mapsto p(t)$ and $t \mapsto \bar{p}\left(t+t_{0}\right)$ solve the same Cauchy problem, and therefore are identical.

We now proceed to case $2 \mathrm{~b}$. In this case, a prospective integral curve is found by limiting $h_{0} \rightarrow \frac{1}{2 c_{1}}\left(c_{0}+c_{1}^{2}\right)$ from the left in case $2 \mathrm{a}$ (Thm. 5.10) and allowing for possible changes in sign.

Proposition 5.14 (case 2b). Suppose $p(\cdot):(-\varepsilon, \varepsilon) \rightarrow \mathfrak{s e}(2)^{*}$ is an integral curve of $\vec{H}$ such that $H(p(0))=$ $h_{0}, C(p(0))=c_{0}>0$ and the conditions of case $2 b$ are satisfied. Then there exists $t_{0} \in \mathbb{R}$ and $\sigma_{1}, \sigma_{2} \in\{-1,1\}$ such that $p(t)=\bar{p}\left(t+t_{0}\right)$ for $t \in(-\varepsilon, \varepsilon)$, where

$$
\left\{\begin{array}{l}
\bar{p}_{1}(t)=c_{1}+\frac{c_{0}-c_{1}^{2}}{c_{1}-\sigma_{1} \sqrt{c_{0}} \cosh (\Omega t)} \\
\bar{p}_{2}(t)=\sqrt{c_{0}} \frac{\sigma_{1} \sigma_{2} \sqrt{c_{0}-c_{1}^{2}} \sinh (\Omega t)}{c_{1}-\sigma_{1} \sqrt{c_{0}} \cosh (\Omega t)} \\
\bar{p}_{3}(t)=\frac{\sigma_{2}\left(c_{0}-c_{1}^{2}\right) \sqrt{\frac{c_{2}}{c_{1}}}}{c_{1}-\sigma_{1} \sqrt{c_{0}} \cosh (\Omega t)} .
\end{array}\right.
$$

Here $\Omega=\sqrt{\frac{c_{0}-c_{1}^{2}}{c_{1} c_{2}}}$. 
Proof. We have $p_{1}(t)^{2}+p_{2}(t)^{2}=c_{0}$ and $p_{1}(t)+\frac{1}{2}\left(\frac{1}{c_{1}} p_{2}(t)^{2}+\frac{1}{c_{2}} p_{3}(t)^{2}\right)=h_{0}$. Hence we have $2 c_{1} p_{1}(t)+$ $p_{2}(t)^{2}-c_{0}-c_{1}^{2}=-\frac{c_{1}}{c_{2}} p_{3}(t)^{2}$. Let $s\left(p_{1}, p_{2}\right)=2 c_{1} p_{1}+p_{2}^{2}-c_{0}-c_{1}^{2}$. Consider the restriction of $s$ to the circle $\mathbb{S}=\left\{\left(p_{1}, p_{2}\right): p_{1}^{2}+p_{2}^{2}=c_{0}\right\} ;\left.s\right|_{\mathbb{S}}$ has exactly two roots $\left(p_{1}, p_{2}\right)=\left(c_{1}, \pm \sqrt{c_{0}-c_{1}^{2}}\right)$.

We claim that $p_{1}(t) \neq c_{1}$ and $p_{3}(t) \neq 0$ for any $t \in(-\varepsilon, \varepsilon)$. Suppose $p_{3}\left(t_{1}\right)=0$ for some $t_{1}$. Then $p_{1}\left(t_{1}\right)=c_{1}$ and so $p\left(t_{1}\right)$ is a equilibrium point. Hence we have $p(\cdot)$ is a constant trajectory, a contradiction. On the other hand suppose $p_{1}\left(t_{1}\right)=c_{1}$ for some $t_{1}$. Then $p_{2}\left(t_{1}\right)^{2}=c_{0}-c_{1}^{2}$ and so $p_{3}\left(t_{1}\right)=0$. Hence $p(\cdot)$ is a constant trajectory, a contradiction. Now $\operatorname{sgn}\left(\bar{p}_{1}(t)-c_{1}\right)=-\sigma_{1}$ and $\operatorname{sgn}\left(\bar{p}_{3}(t)\right)=-\sigma_{1} \sigma_{2}$. Let $\sigma_{1}=-\operatorname{sgn}\left(p_{1}(0)-c_{1}\right)$ and $\sigma_{2}=-\sigma_{1} \operatorname{sgn}\left(p_{3}(0)\right)$.

We claim that there exists $t_{0} \in \mathbb{R}$ such that $p_{2}(0)=\bar{p}_{2}\left(t_{0}\right)$ and $\operatorname{sgn}\left(p_{1}(0)\right)=\operatorname{sgn}\left(\bar{p}_{1}\left(t_{0}\right)\right)$. We need to consider two cases for $p_{1}(0)$. First suppose $p_{1}(0)>c_{1}$. Then $\sigma_{1}=-1$. We have $-\sqrt{c_{0}-c_{1}^{2}}<p_{2}(0)<\sqrt{c_{0}-c_{1}^{2}}$,

$$
\lim _{t \rightarrow-\infty} \bar{p}_{2}(t)=\sigma_{2} \sqrt{c_{0}-c_{1}^{2}} \quad \lim _{t \rightarrow \infty} \bar{p}_{2}(t)=-\sigma_{2} \sqrt{c_{0}-c_{1}^{2}}
$$

and $\bar{p}_{1}(t)>0$ for $t \in \mathbb{R}$. Thus there exists $t_{0} \in \mathbb{R}$ satisfying the claim. Now suppose $p_{1}(0)<c_{1}$. Then $\sigma_{1}=1$. Let $\alpha=\frac{1}{\Omega} \cosh ^{-1}\left(\frac{\sqrt{c_{0}}}{c_{1}}\right)>0$. We have

$$
\begin{aligned}
\bar{p}_{2}(-\alpha) & =\sigma_{2} \sqrt{c_{0}} & \bar{p}_{2}(\alpha) & =-\sigma_{2} \sqrt{c_{0}} \\
\lim _{t \rightarrow-\infty} \bar{p}_{2}(t) & =\sigma_{2} \sqrt{c_{0}-c_{1}^{2}} & \lim _{t \rightarrow \infty} \bar{p}_{2}(t) & =-\sigma_{2} \sqrt{c_{0}-c_{1}^{2}}
\end{aligned}
$$

and

$$
\begin{array}{ll}
\bar{p}_{1}(t)<0 & \text { for } t \in(-\infty,-\alpha) \cup(\alpha, \infty) \\
\bar{p}_{1}(t)>0 & \text { for } t \in(-\alpha, \alpha) .
\end{array}
$$

Note that $-\infty<-\alpha<\alpha<\infty$. We have $-\sqrt{c_{0}} \leq p_{2}(0) \leq \sqrt{c_{0}}$. If $p_{1}(0) \leq 0$ then there exists $t_{0} \in[-\alpha, \alpha]$ satisfying the claim. If $0<p_{1}<c_{1}$, then $-\sqrt{c_{0}}<p_{2}(0)<-\sqrt{c_{0}-c_{1}^{2}}$ or $\sqrt{c_{0}-c_{1}^{2}}<p_{2}(0)<\sqrt{c_{0}}$. In either case there exists $t_{0} \in(-\infty,-\alpha) \cup(\alpha, \infty)$ satisfying the claim.

By using the constant of motion $C$, we find $p_{1}(0)^{2}=\bar{p}_{1}\left(t_{0}\right)^{2}$. However, we have that $\operatorname{sgn}\left(p_{1}(0)\right)=$ $\operatorname{sgn}\left(\bar{p}_{1}\left(t_{0}\right)\right)$ and so $p_{1}(0)=\bar{p}_{1}\left(t_{0}\right)$. Next, by using the constant of motion $H$, we find $p_{3}(0)^{2}=\bar{p}_{3}\left(t_{0}\right)^{2}$. However $\operatorname{sgn}\left(p_{3}(0)\right)=-\sigma_{1} \sigma_{2}=\operatorname{sgn}\left(\bar{p}_{3}\left(t_{0}\right)\right)$ and so $p_{3}(0)=\bar{p}_{3}\left(t_{0}\right)$. Consequently the integral curves $t \mapsto p(t)$ and $t \mapsto \bar{p}\left(t+t_{0}\right)$ solve the same Cauchy problem, and therefore are identical.

Moving on to case $2 \mathrm{c}(\mathrm{i})$, we again find a prospective integral curve by use of formula (2.9).

Theorem 5.15 (case $2 \mathrm{c}(\mathrm{i}))$. Suppose $p(\cdot):(-\varepsilon, \varepsilon) \rightarrow \mathfrak{s e}(2)^{*}$ is an integral curve of $\vec{H}$ such that $H(p(0))=h_{0}$, $C(p(0))=c_{0}>0$ and the conditions of case $2 c(i)$ are satisfied. Then there exists $t_{0} \in \mathbb{R}$ and $\sigma \in\{-1,1\}$ such that $p(t)=\bar{p}\left(t+t_{0}\right)$ for $t \in(-\varepsilon, \varepsilon)$, where

$$
\left\{\begin{array}{l}
\bar{p}_{1}(t)=\left(h_{0}-\delta\right) \frac{\frac{h_{0}+\delta}{h_{0}-\delta} k^{\prime} \sqrt{h_{0}-\delta}-\sigma \sqrt{h_{0}+\delta} \operatorname{dn}(\Omega t, k)}{k^{\prime} \sqrt{h_{0}-\delta}-\sigma \sqrt{h_{0}+\delta} \operatorname{dn}(\Omega t, k)} \\
\bar{p}_{2}(t)=-k \sqrt{2 \delta\left(h_{0}^{2}-\delta^{2}\right)} \frac{\operatorname{cn}(\Omega t, k)}{k^{\prime} \sqrt{h_{0}-\delta}-\sigma \sqrt{h_{0}+\delta} \operatorname{dn}(\Omega t, k)} \\
\bar{p}_{3}(t)=2 \sigma \delta k^{\prime} \sqrt{c_{2}} \frac{\operatorname{sn}(\Omega t, k)}{k^{\prime} \sqrt{h_{0}-\delta}-\sigma \sqrt{h_{0}+\delta} \operatorname{dn}(\Omega t, k)}
\end{array}\right.
$$

Here $\delta=\sqrt{h_{0}^{2}-c_{0}}, \Omega=\sqrt{\frac{\left(h_{0}-\delta\right)\left(h_{0}-c_{1}+\delta\right)}{c_{1} c_{2}}}, k=\sqrt{\frac{2 \delta c_{1}}{\left(h_{0}-\delta\right)\left(h_{0}-c_{1}+\delta\right)}}$ and $k^{\prime}=\sqrt{\frac{\left(h_{0}+\delta\right)\left(h_{0}-c_{1}-\delta\right)}{\left(h_{0}-\delta\right)\left(h_{0}-c_{1}+\delta\right)}}$. 
Proof. We have $p_{1}(0)+\frac{1}{2}\left(\frac{1}{c_{1}} p_{2}(0)^{2}+\frac{1}{c_{2}} p_{3}(0)^{2}\right)=h_{0}$ and $p_{1}(0)^{2}+p_{2}(0)^{2}=c_{0}$. Hence $p_{1}(0)+\frac{1}{2 c_{1}} p_{2}(0)^{2}-h_{0} \leq 0$. Let $s\left(p_{1}, p_{2}\right)=p_{1}+\frac{1}{2 c_{1}} p_{2}^{2}-h_{0}$. Consider the restriction of $s$ to the circle $\mathbb{S}=\left\{\left(p_{1}, p_{2}\right): p_{1}^{2}+p_{2}^{2}=c_{0}\right\} ;\left.s\right|_{\mathbb{S}}$ has exactly four roots $\left(p_{1}, p_{2}\right)=\left(\alpha_{1}, \pm \alpha_{2}\right)$ and $\left(p_{1}, p_{2}\right)=\left(\beta_{1}, \pm \beta_{2}\right)$, where

$$
\begin{array}{ll}
\alpha_{1}=c_{1}+\sqrt{c_{0}+c_{1}^{2}-2 c_{1} h_{0}} & \alpha_{2}=\sqrt{2 c_{1}} \sqrt{h_{0}-c_{1}-\sqrt{c_{0}+c_{1}^{2}-2 c_{1} h_{0}}} \\
\beta_{1}=c_{1}-\sqrt{c_{0}+c_{1}^{2}-2 c_{1} h_{0}} & \beta_{2}=\sqrt{2 c_{1}} \sqrt{h_{0}-c_{1}+\sqrt{c_{0}+c_{1}^{2}-2 c_{1} h_{0}} .}
\end{array}
$$

Consequently, if $p_{1}(0)>c_{1}$, then $\alpha_{1} \leq p_{1}(0) \leq \sqrt{c_{0}}$. On the other hand, if $p_{1}(0)<c_{1}$, then $-\sqrt{c_{0}} \leq p_{1}(0) \leq \beta_{1}$.

It is easy to show, under the conditions of $2 c(\mathrm{i})$, that $p_{1}(0) \neq c_{1}$. Let $\sigma=-\operatorname{sgn}\left(p_{1}(0)-c_{1}\right)$. Suppose $p_{1}(0)>c_{1}$, i.e., $\sigma=-1$. A somewhat involved computation yields $\bar{p}_{1}(0)=\alpha_{1}$ and $\bar{p}_{1}\left(\frac{K}{\Omega}\right)=\sqrt{c_{0}}$. Hence there exists $t_{1} \in\left[0, \frac{K}{\Omega}\right]$ such that $\bar{p}_{1}\left(t_{1}\right)=p_{1}(0)$. On the other hand, suppose $p_{1}(0)<c_{1}$, i.e., $\sigma=1$. Again, a somewhat involved computation yields $\bar{p}_{1}(0)=\beta_{1}$ and $\bar{p}_{1}\left(\frac{K}{\Omega}\right)=-\sqrt{c_{0}}$. Hence there again exists $t_{1} \in\left[0, \frac{K}{\Omega}\right]$ such that $\bar{p}_{1}\left(t_{1}\right)=p_{1}(0)$.

Finally, we claim that there exists $t_{0} \in \mathbb{R}$ such that $\bar{p}\left(t_{0}\right)=p(0)$. By using the constants of motion $C$ and $H$, we get $p_{2}(0)= \pm \bar{p}_{2}\left(t_{1}\right)$ and $p_{3}(0)= \pm \bar{p}_{3}\left(t_{1}\right)$. Now

$$
\begin{aligned}
& \bar{p}_{1}(-t)=\bar{p}_{1}(t) \quad \bar{p}_{1}\left(t+\frac{2 K}{\Omega}\right)=\bar{p}_{1}(t) \quad \bar{p}_{1}\left(-t+\frac{2 K}{\Omega}\right)=\bar{p}_{1}(t) \\
& \bar{p}_{2}(-t)=\bar{p}_{2}(t) \quad \bar{p}_{2}\left(t+\frac{2 K}{\Omega}\right)=-\bar{p}_{2}(t) \quad \bar{p}_{2}\left(-t+\frac{2 K}{\Omega}\right)=-\bar{p}_{2}(t) \\
& \bar{p}_{3}(-t)=-\bar{p}_{3}(t) \quad \bar{p}_{3}\left(t+\frac{2 K}{\Omega}\right)=-\bar{p}_{3}(t) \quad \bar{p}_{3}\left(-t+\frac{2 K}{\Omega}\right)=\bar{p}_{3}(t) .
\end{aligned}
$$

Therefore there exists $t_{0} \in \mathbb{R}\left(t_{0}=t_{1}, t_{0}=-t_{1}, t_{0}=t_{1}+\frac{2 K}{\Omega}\right.$, or $\left.t_{0}=-t_{1}+\frac{2 K}{\Omega}\right)$ such that $p(0)=\bar{p}\left(t_{0}\right)$. Consequently the integral curves $t \mapsto p(t)$ and $t \mapsto \bar{p}\left(t+t_{0}\right)$ solve the same Cauchy problem, and therefore are identical.

We are left to consider case 2c(ii). By limiting $c_{0} \rightarrow h_{0}^{2}$ in case 2c(iii) (Thm. 5.13) and allowing for possible changes in sign, we obtain a prospective integral curve.

Proposition 5.16 (case $2 \mathrm{c}(\mathrm{ii}))$. Suppose $p(\cdot):(-\varepsilon, \varepsilon) \rightarrow \mathfrak{s e}(2)^{*}$ is an integral curve of $\vec{H}$ such that $H(p(0))=$ $h_{0}, C(p(0))=c_{0}>0$ and the conditions of case $2 c(i i)$ are satisfied. Then there exists $t_{0} \in \mathbb{R}$ such that $p(t)=\bar{p}\left(t+t_{0}\right)$ for $t \in(-\varepsilon, \varepsilon)$, where

$$
\left\{\begin{array}{l}
\bar{p}_{1}(t)=-\sqrt{c_{0}} \frac{\sqrt{c_{0}}+\frac{1}{2} c_{1}(-3+\cos (2 \Omega t))}{\sqrt{c_{0}}-\frac{1}{2} c_{1}(1+\cos (2 \Omega t))} \\
\bar{p}_{2}(t)=\frac{2 \sqrt{c_{0}\left(\sqrt{c_{0}}-c_{1}\right) c_{1}} \sin (\Omega t)}{\sqrt{c_{0}}-\frac{1}{2} c_{1}(1+\cos (2 \Omega t))} \\
\bar{p}_{3}(t)=\frac{2\left(\sqrt{c_{0}}-c_{1}\right) \sqrt{\sqrt{c_{0}} c_{2}} \cos (\Omega t)}{\sqrt{c_{0}}-\frac{1}{2} c_{1}(1+\cos (2 \Omega t))} .
\end{array}\right.
$$

Here $\Omega=\sqrt{\frac{\sqrt{c_{0}}\left(\sqrt{c_{0}}-c_{1}\right)}{c_{1} c_{2}}}$.

Proof. We have $p_{1}(0)+\frac{1}{2}\left(\frac{1}{c_{1}} p_{2}(0)^{2}+\frac{1}{c_{2}} p_{3}(0)^{2}\right)=h_{0}$ and $p_{1}(0)^{2}+p_{2}(0)^{2}=c_{0}$. Hence $p_{1}(0)+\frac{1}{2 c_{1}} p_{2}(0)^{2}-h_{0} \leq 0$. Let $s\left(p_{1}, p_{2}\right)=p_{1}+\frac{1}{2 c_{1}} p_{2}^{2}-h_{0}$. Consider the restriction of $s$ to the circle $\mathbb{S}=\left\{\left(p_{1}, p_{2}\right): p_{1}^{2}+p_{2}^{2}=c_{0}\right\} ;\left.s\right|_{\mathbb{S}}$ has exactly three roots

$$
\left(p_{1}, p_{2}\right)=\left(\sqrt{c_{0}}, 0\right) \quad\left(p_{1}, p_{2}\right)=\left(2 c_{1}-\sqrt{c_{0}}, \pm 2 \sqrt{c_{1}} \sqrt{\sqrt{c_{0}}-c_{1}}\right) .
$$


Consequently $p_{1}(0)=\sqrt{c_{0}}$ or $-\sqrt{c_{0}} \leq p_{1}(0) \leq 2 c_{1}-\sqrt{c_{0}}$. However, if $p_{1}(0)=\sqrt{c_{0}}$, then $p_{2}(0)=0$ and $p_{3}(0)=0$, i.e., $p(\cdot)$ is a constant trajectory. Thus this case can be discarded.

Now $\bar{p}_{1}(0)=-\sqrt{c_{0}}$ and $\bar{p}_{1}\left(\frac{\pi}{2 \Omega}\right)=2 c_{1}-\sqrt{c_{0}}$. Thus there exists $t_{1} \in\left[0, \frac{\pi}{2 \Omega}\right]$ such that $\bar{p}_{1}\left(t_{1}\right)=p_{1}(0)$. By using the constants of motion $C$ and $H$, we get $p_{2}(0)= \pm \bar{p}_{2}\left(t_{1}\right)$ and $p_{3}(0)= \pm \bar{p}_{3}\left(t_{1}\right)$. Now

$$
\begin{array}{lll}
\bar{p}_{1}(-t)=\bar{p}_{1}(t) & \bar{p}_{1}\left(t+\frac{\pi}{\Omega}\right)=\bar{p}_{1}(t) & \bar{p}_{1}\left(-t+\frac{\pi}{\Omega}\right)=\bar{p}_{1}(t) \\
\bar{p}_{2}(-t)=-\bar{p}_{2}(t) & \bar{p}_{2}\left(t+\frac{\pi}{\Omega}\right)=-\bar{p}_{2}(t) & \bar{p}_{2}\left(-t+\frac{\pi}{\Omega}\right)=\bar{p}_{2}(t) \\
\bar{p}_{3}(-t)=\bar{p}_{3}(t) & \bar{p}_{3}\left(t+\frac{\pi}{\Omega}\right)=-\bar{p}_{3}(t) & \bar{p}_{3}\left(-t+\frac{\pi}{\Omega}\right)=-\bar{p}_{3}(t) .
\end{array}
$$

Therefore there exists $t_{0} \in \mathbb{R}\left(t_{0}=t_{1}, t_{0}=-t_{1}, t_{0}=t_{1}+\frac{\pi}{\Omega}\right.$, or $\left.t_{0}=-t_{1}+\frac{\pi}{\Omega}\right)$ such that $p(0)=\bar{p}\left(t_{0}\right)$. Consequently the integral curves $t \mapsto p(t)$ and $t \mapsto \bar{p}\left(t+t_{0}\right)$ solve the same Cauchy problem, and therefore are identical.

Finally, let us consider the invariant control problem LiCP(3). There is only one typical case which is easy to integrate. Again, we graph the level sets of $H$ and $C$ and their intersection.
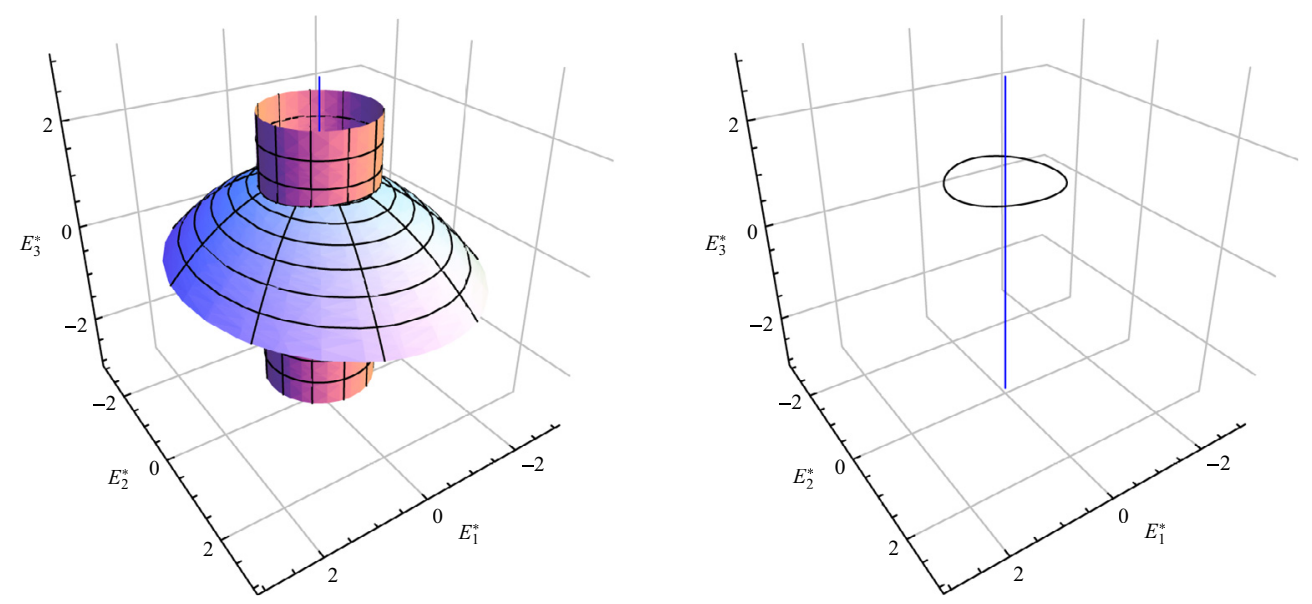

Figure 3. Typical reduced extremal of $\operatorname{LiCP}(3)$.

Proposition 5.17. The reduced Hamilton equations (3.6) have the solutions

$$
\left\{\begin{array}{l}
p_{1}(t)=\sqrt{c_{0}} \sin \left(\alpha t+t_{0}\right) \\
p_{2}(t)=\sqrt{c_{0}} \cos \left(\alpha t+t_{0}\right) \\
p_{3}(t)=\frac{h_{0}}{\alpha}-\frac{c_{0}}{2 \alpha}\left(\frac{1}{c_{1}} \sin ^{2}\left(\alpha t+t_{0}\right)+\frac{1}{c_{2}} \cos ^{2}\left(\alpha t+t_{0}\right)\right)
\end{array}\right.
$$

where $c_{0}=C(p(0)), h_{0}=H(p(0))$ and $t_{0} \in \mathbb{R}$. 


\section{Conclusion}

This paper shows that there are only three types of two-input left-invariant control affine systems on SE (2) (up to equivalence), and then studies a general optimal control problem (with quadratic cost) for each type. In each case the problem is reduced (via the Pontryagin Maximum Principle) to the study of a single Hamiltonian $H$ on the Poisson space $\mathfrak{s e}(2)_{-}^{*}$. The stability nature of all equilibrium states (of the reduced system) is investigated by use of the energy-Casimir method. $\vec{H}$ is shown to be complete. Also, every maximal integral curve is shown to be a constant, periodic or bounded curve.

For each problem, explicit expressions for all integral curves of $\vec{H}$ are found, up to a choice of sign and a translation in the independent variable. This is achieved (in most instances) by reducing the given system of differential equations to a single (separable) differential equation and then transforming it into standard form. Thereafter, an application of an appropriate integral formula yields a solution involving Jacobi elliptic functions. Using the constants of motion $C$ and $H$ (and allowing for possible changes in sign) an explicit expression for an integral curve of $\vec{H}$ is then obtained. Finally, we verify that any other integral curve, satisfying the same partitioning conditions, is identical to the one produced (up to a translation).

We now have explicit expressions for all (normal) extremal controls. A natural next step would be to solve the equations on the base SE (2), i.e., to obtain expressions for the (normal) extremal trajectories. For the control problem LiCP(1), this was essentially accomplished in [26]. The inhomogeneous case has yet to be considered.

Single-input systems evolving on SE (2) have been considered in [2]. Specifically, it was shown that there are only two typical cases (up to equivalence). Likewise, stability and explicit integration were addressed. The three-input case is a topic for future research.

\section{Appendix A. Tabulation of integral CURVES}

TABLE A.1. Integral curves of $\vec{H}$ for reduced extremals of LiCP(1) and LiCP(3).

\begin{tabular}{|lll|}
\hline Case a & & $c_{0}>2 c_{1} h_{0}$ \\
\hline $\bar{p}_{1}(t)=\sigma \sqrt{c_{0}} \operatorname{dn}(\Omega t, k)$ & $k=\sqrt{\frac{2 c_{1} h_{0}}{c_{0}}}$ \\
$\bar{p}_{2}(t)=\sqrt{2 c_{1} h_{0}} \operatorname{sn}(\Omega t, k)$ & $\Omega=\sqrt{\frac{c_{0}}{c_{1} c_{2}}}$ \\
$\bar{p}_{3}(t)=-\sigma \sqrt{2 c_{2} h_{0}} \operatorname{cn}(\Omega t, k)$ & $\sigma \in\{-1,1\}$ \\
\hline \hline Case b & $c_{0}=2 c_{1} h_{0}$ \\
\hline $\bar{p}_{1}(t)=\sigma_{1} \sigma_{2} \sqrt{c_{0}} \operatorname{sech}(\Omega t)$ & $\Omega=\sqrt{\frac{c_{0}}{c_{1} c_{2}}}$ \\
$\bar{p}_{2}(t)=\sigma_{1} \sqrt{c_{0}} \tanh (\Omega t)$ & $\sigma_{1} \in\{-1,1\}$ \\
$\bar{p}_{3}(t)=-\sigma_{2} \sqrt{\frac{c_{0} c_{2}}{c_{1}}} \operatorname{sech}(\Omega t)$, & $\sigma_{2} \in\{-1,1\}$ \\
\hline \hline Case c & & \\
\hline $\bar{p}_{1}(t)=\sigma \sqrt{c_{0}} \operatorname{cn}(\Omega t, k)$ & $k=\sqrt{\frac{c_{0}}{2 c_{1} h_{0}}}$ \\
$\bar{p}_{2}(t)=\sqrt{c_{0}} \operatorname{sn}(\Omega t, k)$ & $\Omega=\sqrt{\frac{2 h_{0}}{c_{2}}}$ \\
$\bar{p}_{3}(t)=-\sigma \sqrt{2 c_{2} h_{0}} \operatorname{dn}(\Omega t, k)$ & \\
\hline \hline $\mathrm{LiCP}(3)$ & \\
\hline$p_{1}(t)=\sqrt{c_{0}} \sin \left(\alpha t+t_{0}\right)$ & \\
$p_{2}(t)=\sqrt{c_{0}} \cos \left(\alpha t+t_{0}\right)$ & \\
$p_{3}(t)=\frac{h_{0}}{\alpha}-\frac{c_{0}}{2 \alpha}\left(\frac{1}{c_{1}} \sin ^{2}\left(\alpha t+t_{0}\right)+\frac{1}{c_{2}} \cos ^{2}\left(\alpha t+t_{0}\right)\right)$ \\
\hline
\end{tabular}


TABLE A.2. Integral curves of $\vec{H}$ for reduced extremals of LiCP(2).

\begin{tabular}{|c|c|}
\hline$c_{0}-c_{1}^{2} \leq 0, \quad h_{0}$ & $-c_{0}$ \\
\hline $\begin{array}{l}\bar{p}_{1}(t)=\sqrt{c_{0}} \frac{\sqrt{h_{0}-\delta}-\sqrt{h_{0}+\delta} \operatorname{sn}(\Omega t, k)}{\sqrt{h_{0}+\delta}-\sqrt{h_{0}-\delta} \operatorname{sn}(\Omega t, k)} \\
\bar{p}_{2}(t)=-\sigma \sqrt{2 c_{0} \delta} \frac{\operatorname{cn}(\Omega t, k)}{\sqrt{h_{0}+\delta}-\sqrt{h_{0}-\delta} \operatorname{sn}(\Omega t, k)} \\
\bar{p}_{3}(t)=\frac{2 \sigma \delta \sqrt{c_{2}}}{k^{\prime}} \frac{\operatorname{dn}(\Omega t, k)}{\sqrt{h_{0}+\delta}-\sqrt{h_{0}-\delta} \operatorname{sn}(\Omega t, k)}\end{array}$ & $\begin{aligned} \delta & =\sqrt{h_{0}^{2}-c_{0}} \\
\Omega & =\sqrt{\frac{\left(h_{0}+\delta\right)\left(c_{1}-h_{0}+\delta\right)}{c_{1} c_{2}}} \\
k & =\sqrt{\frac{\left(h_{0}-\delta\right)\left(c_{1}-h_{0}-\delta\right)}{\left(h_{0}+\delta\right)\left(c_{1}-h_{0}+\delta\right)}} \\
\sigma & \in\{-1,1\}\end{aligned}$ \\
\hline
\end{tabular}

\begin{tabular}{|lcc|}
\hline \hline Case 1a(ii) $\quad c_{0}-c_{1}^{2} \leq 0$, & $h_{0}>\sqrt{c_{0}}$, & $c_{1}-h_{0}=\sqrt{h_{0}^{2}-c_{0}}$ \\
\hline $\bar{p}_{1}(t)=\sqrt{c_{0}} \frac{\sqrt{h_{0}-\delta}-\sqrt{h_{0}+\delta} \sin (\Omega t)}{\sqrt{h_{0}+\delta}-\sqrt{h_{0}-\delta} \sin (\Omega t)}$ & $\vdots$ & $\delta=\sqrt{h_{0}^{2}-c_{0}}$ \\
$\bar{p}_{2}(t)=-\sigma \sqrt{2 c_{0} \delta} \frac{\cos (\Omega t)}{\sqrt{h_{0}+\delta}-\sqrt{h_{0}-\delta} \sin (\Omega t)}$ & $\Omega=\sqrt{\frac{2\left(c_{1}-h_{0}\right)}{c_{2}}}$ \\
$\bar{p}_{3}(t)=\frac{2 \sigma \delta \sqrt{c_{2}}}{\sqrt{h_{0}+\delta}-\sqrt{h_{0}-\delta} \sin (\Omega t)}$ & $\vdots$ & $\sigma \in\{-1,1\}$ \\
\hline \hline
\end{tabular}

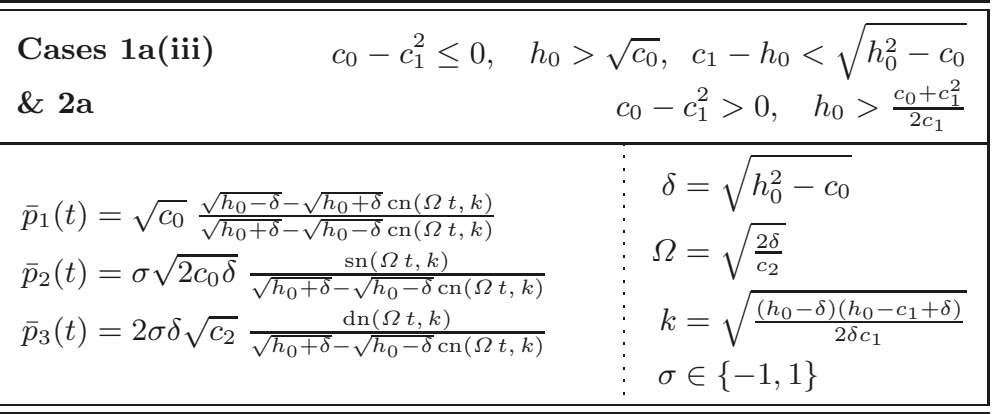

\begin{tabular}{lll}
\hline Case 1b(i) & $c_{0}-c_{1}^{2} \leq 0$, & $h_{0}=\sqrt{c_{0}}, \quad h_{0}<c_{1}$ \\
\hline $\bar{p}_{1}(t)=\frac{h_{0}}{2} \frac{c_{1}(\cosh (2 \Omega t)-3)+2 h_{0}}{c_{1} \cosh (\Omega t)-h_{0}}$ & $\vdots$ & $\Omega=\sqrt{\frac{h_{0}\left(c_{1}-h_{0}\right)}{c_{1} c_{2}}}$ \\
$\bar{p}_{2}(t)=2 \sigma h_{0} \sqrt{c_{1}\left(c_{1}-h_{0}\right)} \frac{\sinh (\Omega t)}{c_{1} \cosh ^{2}(\Omega t)-h_{0}}$ & $\quad$ & $\sigma \in\{-1,1\}$ \\
$\bar{p}_{3}(t)=2 \sigma\left(c_{1}-h_{0}\right) \sqrt{c_{2} h_{0}} \frac{\cosh ^{2}(\Omega t)}{c_{1} \cosh ^{2}(\Omega t)-h_{0}}$ & \\
\hline \hline
\end{tabular}

\begin{tabular}{|lcl|}
\hline \hline Case 1b(ii) & $c_{0}-c_{1}^{2} \leq 0, \quad h_{0}=\sqrt{c_{0}}, \quad h_{0}=c_{1}$ \\
\hline $\bar{p}_{1}(t)=c_{1} \frac{c_{1} t^{2}-c_{2}}{c_{1} t^{2}+c_{2}}$ & $\vdots$ & \\
$\bar{p}_{2}(t)=2 \sigma c_{1} \sqrt{c_{1} c_{2}} \frac{t}{c_{1} t^{2}+c_{2}}$ & & $\sigma \in\{-1,1\}$ \\
$\bar{p}_{3}(t)=2 \sigma c_{2} \sqrt{c_{1} c_{2}} \frac{1}{c_{1} t^{2}+c_{2}}$ & $\vdots$ & \\
\hline
\end{tabular}


TABLE A.3. Integral curves of $\vec{H}$ for reduced extremals of LiCP(2) (continued).

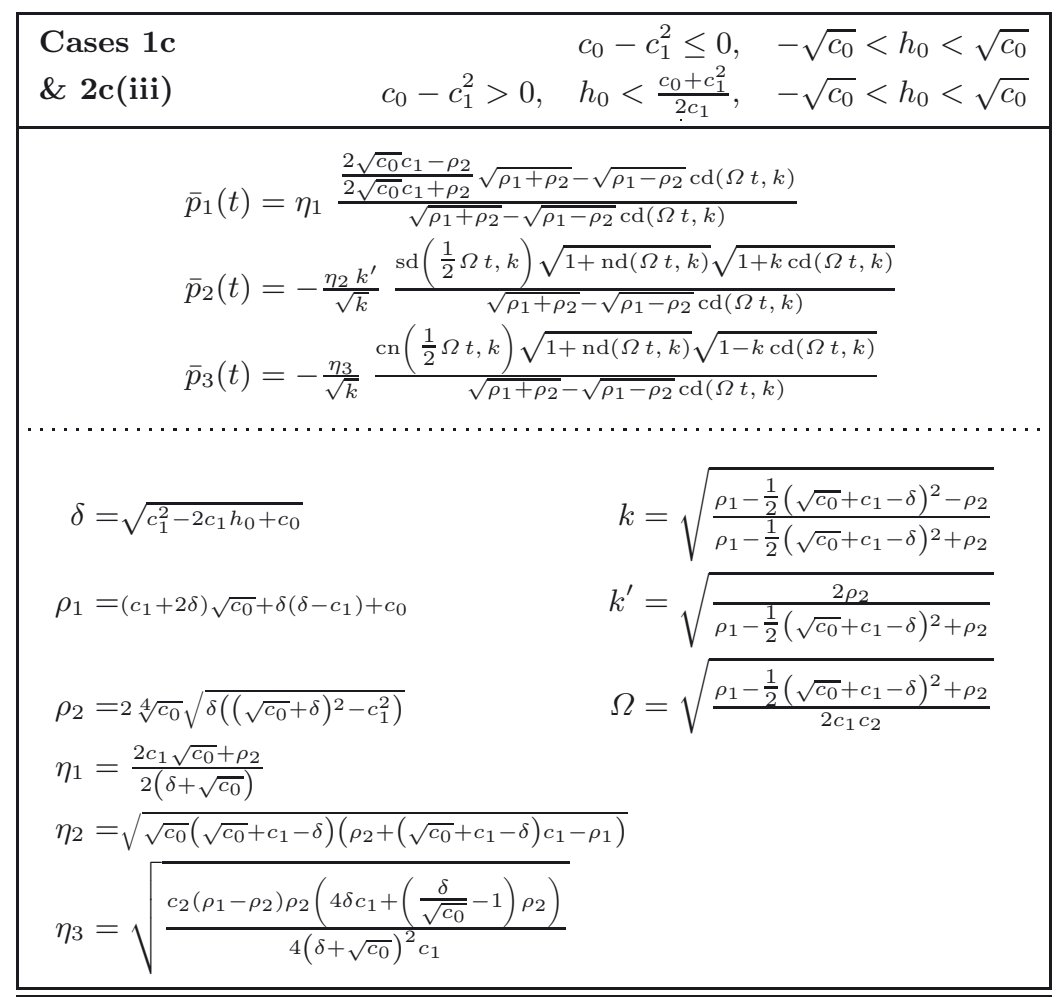

\begin{tabular}{|c|c|}
\hline Case 2b & $c_{0}-c_{1}^{2}>0, \quad h_{0}=\frac{c_{0}+c_{1}^{2}}{2 c_{1}}$ \\
\hline $\begin{array}{l}\bar{p}_{1}(t)=c_{1}+\frac{c_{0}-c_{1}^{2}}{c_{1}-\sigma_{1} \sqrt{c_{0}} \cosh (\Omega t)} \\
\bar{p}_{2}(t)=\sqrt{c_{0}} \frac{\sigma_{1} \sigma_{2} \sqrt{c_{0}-c_{1}^{2}} \sinh (\Omega t)}{c_{1}-\sigma_{1} \sqrt{c_{0}} \cosh (\Omega t)} \\
\bar{p}_{3}(t)=\frac{\sigma_{2}\left(c_{0}-c_{1}^{2}\right) \sqrt{\frac{c_{2}}{c_{1}}}}{c_{1}-\sigma_{1} \sqrt{c_{0}} \cosh (\Omega t)}\end{array}$ & $\begin{aligned} \Omega & =\sqrt{\frac{c_{0}-c_{1}{ }^{2}}{c_{1} c_{2}}} \\
\sigma_{1} & \in\{-1,1\} \\
\sigma_{2} & \in\{-1,1\}\end{aligned}$ \\
\hline
\end{tabular}

\begin{tabular}{|c|c|}
\hline$c_{0}-c_{1}^{2}>0$ & $h_{0}<\frac{c_{0}+c_{1}^{2}}{2 c_{1}}, \quad h_{0}>\sqrt{c_{0}}$ \\
\hline $\begin{array}{l}\bar{p}_{1}(t)=\frac{k^{\prime}\left(h_{0}+\delta\right) \sqrt{h_{0}-\delta}-\sigma\left(h_{0}-\delta\right) \sqrt{h_{0}+\delta} \operatorname{dn}(\Omega t, k)}{k^{\prime} \sqrt{h_{0}-\delta}-\sigma \sqrt{h_{0}+\delta} \operatorname{dn}(\Omega t, k)} \\
\bar{p}_{2}(t)=-\frac{k \sqrt{2 \delta\left(h_{0}^{2}-\delta^{2}\right)} \operatorname{cn}(\Omega t, k)}{k^{\prime} \sqrt{h_{0}-\delta}-\sigma \sqrt{h_{0}+\delta} \operatorname{dn}(\Omega t, k)} \\
\bar{p}_{3}(t)=\frac{2 \sigma \delta k^{\prime} \sqrt{c_{2}} \operatorname{sn}(\Omega t, k)}{k^{\prime} \sqrt{h_{0}-\delta}-\sigma \sqrt{h_{0}+\delta} \operatorname{dn}(\Omega t, k)}\end{array}$ & $\begin{aligned} \delta & =\sqrt{h_{0}^{2}-c_{0}} \\
\Omega & =\sqrt{\frac{\left(h_{0}-\delta\right)\left(h_{0}-c_{1}+\delta\right)}{c_{1} c_{2}}} \\
k & =\sqrt{\frac{2 \delta c_{1}}{\left(h_{0}-\delta\right)\left(h_{0}-c_{1}+\delta\right)}} \\
\sigma & \in\{-1,1\}\end{aligned}$ \\
\hline
\end{tabular}

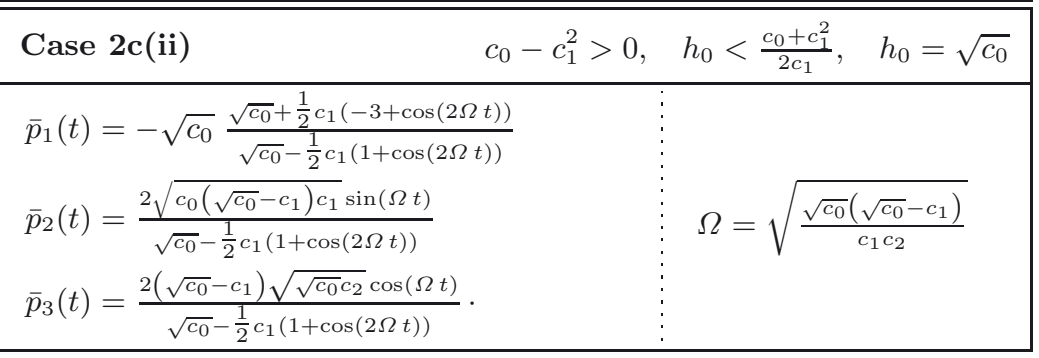




\section{REFERENCES}

[1] R. Abraham and J.E. Marsden, Foundations of Mechanics, 2nd edition. Addison-Wesley (1978).

[2] R.M. Adams, R. Biggs and C.C. Remsing, Single-input control systems on the Euclidean group SE (2). Eur. J. Pure Appl. Math. 5 (2012) 1-15.

[3] A.A. Agrachev and Y.L. Sachkov, Control Theory from the Geometric Viewpoint. Springer-Verlag (2004).

[4] J.V. Armitage and W.F. Eberlein, Elliptic Functions. Cambridge University Press (2006).

[5] R. Biggs and C.C. Remsing, A category of control systems. An. Şt. Univ. Ovidius Constanţa 20 (2012) 355-368.

[6] R. Biggs and C.C. Remsing, On the equivalence of control systems on Lie groups. Publ. Math. Debrecen (submitted).

[7] R. Biggs and C.C. Remsing, On the equivalence of cost-extended control systems on Lie groups. Proc. 8th WSEAS Int. Conf. Dyn. Syst. Control. Porto, Portugal (2012) 60-65.

[8] R.W. Brockett, System theory on group manifolds and coset spaces. SIAM J. Control 10 (1972) 265-284.

[9] D.D. Holm, J.E. Marsden, T. Ratiu and A. Weinstein, Nonlinear stability of fluid and plasma equilibria. Phys. Rep. 123 (1985) $1-116$.

[10] V. Jurdjevic, Non-Euclidean elastica. Amer. J. Math. 117 (1995) 93-124.

[11] V. Jurdjevic, Geometric Control Theory. Cambridge University Press (1997).

[12] V. Jurdjevic, Optimal control problems on Lie groups: crossroads between geometry and mechanics, in Geometry of Feedback and Optimal Control, edited by B. Jakubczyk and W. Respondek, M. Dekker (1998) 257-303.

[13] V. Jurdjevic and H.J. Sussmann, Control systems on Lie groups. J. Differ. Equ. 12 (1972) 313-329.

[14] P.S. Krishnaprasad, Optimal control and Poisson reduction, Technical Research Report T.R.93-87. Inst. Systems Research, University of Maryland (1993).

[15] D.F. Lawden, Elliptic Functions and Applications. Springer-Verlag (1989).

[16] J.E. Marsden and T.S. Ratiu, Introduction to Mechanics and Symmetry. 2nd edition. Springer-Verlag (1999).

[17] I. Moiseev and Y.L. Sachkov, Maxwell strata in sub-Riemannian problem on the group of motions of a plane. ESAIM: COCV 16 (2010) 380-399.

[18] J-P. Ortega and T.S. Ratiu, Non-linear stability of singular relative periodic orbits in Hamiltonian systems with symmetry. J. Geom. Phys. 32 (1999) 160-188.

[19] J-P. Ortega, V. Planas-Bielsa and T.S. Ratiu, Asymptotic and Lyapunov stability of constrained and Poisson equilibria. J. Differ. Equ. 214 (2005) 92-127.

[20] L. Perko, Differential Equations and Dynamical Systems, 3rd edition. Springer-Verlag (2001).

[21] M. Puta, Hamiltonian Mechanical Systems and Geometric Quantization. Kluwer (1993).

$[22]$ M. Puta, S. Chirici and A. Voitecovici, An optimal control problem on the Lie group SE (2, R). Publ. Math. Debrecen 60 (2002) 15-22.

[23] M. Puta, G. Schwab and A. Voitecovici, Some remarks on an optimal control problem on the Lie group SE (2, R $)$. An. Şt. Univ. A.I. Cuza Iaşi, ser. Mat. 49 (2003) 249-256.

[24] C.C. Remsing, Optimal control and Hamilton-Poisson formalism. Int. J. Pure Appl. Math. 59 (2010) 11-17.

[25] C.C. Remsing, Control and stability on the Euclidean group SE (2). Lect. Notes Eng. Comput. Sci. Proc. WCE 2011. London, UK, 225-230.

[26] Y.L. Sachkov, Maxwell strata in the Euler elastic problem. J. Dyn. Control Syst. 14 (2008) 169-234.

[27] Y.L. Sachkov, Conjugate and cut time in the sub-Riemannian problem on the group of motions of a plane. ESAIM: COCV 16 (2010) 1018-1039.

[28] Y.L. Sachkov, Cut locus and optimal synthesis in the sub-Riemannian problem on the group of motions of a plane. ESAIM: COCV 17 (2011) 293-321. 